\title{
New insights on the role of organic speciation in the biogeochemical cycle of dissolved cobalt in the southeastern Atlantic and the Southern Ocean
}

\author{
J. Bown, M. Boye, and D. M. Nelson \\ Institut Universitaire Européen de la Mer (IUEM), UMS3113 - Laboratoire des Sciences de l'Environnement Marin, \\ UMR6539, Technopôle Brest Iroise, Place Nicolas Copernic, 29280 Plouzané, France
}

Correspondence to: J. Bown (johann.bown@univ-brest.fr)

Received: 11 February 2012 - Published in Biogeosciences Discuss.: 16 March 2012

Revised: 16 June 2012 - Accepted: 24 June 2012 - Published: 27 July 2012

\begin{abstract}
The organic speciation of dissolved cobalt (DCo) was investigated in the subtropical region of the southeastern Atlantic, and in the Southern Ocean in the Antarctic Circumpolar Current (ACC) and the northern Weddell Gyre, between $34^{\circ} 25^{\prime} \mathrm{S}$ and $57^{\circ} 33^{\prime} \mathrm{S}$ along the Greenwich Meridian during the austral summer of 2008. The organic speciation of dissolved cobalt was determined by competing ligand exchange adsorptive cathodic stripping voltammetry (CLEAdCSV) using nioxime as a competing ligand. The concentrations of the organic ligands (L) ranged between 26 and $73 \mathrm{pM}$, and the conditional stability constants $\left(\log \mathrm{K}^{\prime} \mathrm{CoL}\right)$ of the organic complexes of Co between 17.9 and 20.1. Most dissolved cobalt was organically complexed in the water-column (60 to $>99.9 \%$ ). There were clear vertical and meridional patterns in the distribution of $\mathrm{L}$ and the organic speciation of DCo along the section. These patterns suggest a biological source of the organic ligands in the surface waters of the subtropical domain and northern subantarctic region, potentially driven by the cyanobacteria, and a removal of the organic Co by direct or indirect biological uptake. The highest L:DCo ratio $\left(5.81 \pm 1.07 \mathrm{pM} \mathrm{pM}^{-1}\right)$ observed in these surface waters reflected the combined effects of ligand production and DCo consumption. As a result of these combined effects, the calculated concentrations of inorganic Co ([Co'] $]$ were very low in the subtropical and subantarctic surface waters, generally between $10^{-19}$ and $10^{-17} \mathrm{M}$. In intermediate and deep waters, the South African margins can be a source of organic ligands, as it was suggested to be for DCo (Bown et al., 2011), although a significant portion of DCo (up to $15 \%$ ) can be stabilized and transported as in-
\end{abstract}

organic species in those DCo-enriched water-masses. Contrastingly, the distribution of L does not suggest an intense biological production of $\mathrm{L}$ around the Antarctic Polar Front where a diatom bloom had recently occurred. Here [Co'] can be several orders of magnitude higher than those reported in the subtropical domain, suggesting that cobalt limitation was unlikely in the ACC domain. The almost invariant L:DCo ratio of $\sim 1$ recorded in these surface waters also reflected the conservative behaviours of both $\mathrm{L}$ and DCo. In deeper waters higher ligand concentrations were observed in waters previously identified as DCo sources (Bown et al., 2011). At those depths the eastward increase of DCo from the Drake Passage to the Greenwich Meridian could be associated with a large scale transport and remineralisation of DCo as organic complexes; here, the fraction stabilized as inorganic Co was also significant (up to $25 \%$ ) in the low oxygenated Upper Circumpolar Deep Waters. Organic speciation may thus be a central factor in the biogeochemical cycle of DCo in those areas, playing a major role in the bioavailability and the geochemistry of Co.

\section{Introduction}

Cobalt can play an important physiological role in phytoplankton metabolism and growth, as it is involved in the structure of the vitamin cobalamin $\left(\mathrm{B}_{12}\right)$ and in the activity of carbonic anhydrase and hydrolytic enzymes (Morel et al., 2003). The vertical distribution of dissolved cobalt (DCo) can range from nutrient-like as found in oligotrophic regions 
of the Atlantic Ocean (Bown et al., 2011; Jakuba et al., 2008; Saito and Moffett, 2001) or in the Ross Sea (Saito et al., 2010) to scavenged-like as found in the North Pacific (Knauer et al., 1982) or conservative like in the Southern Ocean (Bown et al., 2011). In surface waters of the Atlantic and the Southern Ocean, DCo concentrations can be low (5-25 pM, Bown et al., 2011; Ellwood et al., 2005; Saito and Moffett, 2001; Saito et al., 2010), and such low concentrations can impact the phytoplankton growth. Recent field observation evidenced co-limitation of diatoms and Phaeocystis antarctica sp. growth by iron and cobalt contained in vitamin $B_{12}$ in the Ross Sea during early austral summer (Bertrand et al., 2007). In that latter study, the colimitation could be related to the absence of vitamin $\mathrm{B}_{12}$ produced by bacteria and archaea during austral spring (Saito et al., 2010), or to a decrease in the biosynthesis of vitamin $\mathrm{B}_{12}$ due to low cobalt concentrations (Gordon and Sullivan, 2008).

Organic complexation likely plays a significant role in the biogeochemical cycle of DCo in the ocean, affecting both its bioavailability (Ellwood and van den Berg, 2001; Ellwood et al., 2005; Saito and Moffett, 2001; Saito et al., 2004) and its solubility in oxygenated seawater (Moffett and Ho, 1996). Electrochemical methods have been used to study the potential affinity of cobalt with dissolved organic matter, and also allow the determination of the total concentration of organic Co-binding ligands (L) and conditional stability constants of the organic complexes $\left(\mathrm{K}^{\prime}{ }_{\mathrm{CoL}}\right)$ (Ellwood et al., 2005; Saito and Moffett, 2001; Saito et al., 2005; Yang and Van den Berg, 2009). Such measurements can lead to improve understanding of the biogeochemical cycle of Co in the open ocean. However, studies of the organic speciation of DCo in the ocean are still scarce. The ocean distribution of Co-binding organic ligands exhibits relative maxima or minima in the euphotic layer (Ellwood and van den Berg, 2001; Saito and Moffett, 2001), with a rather uniform distribution or increasing concentrations in deeper waters (Ellwood and van den Berg, 2001; Ellwood et al., 2005). Differences in the distribution and concentration of $\mathrm{L}$ probably reflect the spatial variety of their sources and sinks in different ocean waters as well as the seasonal variability of these terms. As far as we know, no data have previously been reported for Co-binding ligand concentrations in deep waters below $2000 \mathrm{~m}$.

The few studies on the organic speciation of cobalt have shown that DCo occurs predominantly as strong organic complexes in the euphotic layer of subtropical ocean regions (Noble et al., 2008; Saito and Moffett, 2001; Saito et al., 2004, 2005). The sources of these ligands are not well characterized, but the release of Co-binding ligands by cyanobacteria has been suggested from laboratory and field studies (Saito and Moffett, 2001; Saito et al., 2002, 2005). Conversely, elevated ligand concentrations have been reported at the depth of the chlorophyll $a$ maximum in the Southern Ocean, south of the Polar Front where cyanobacteria were absent (Ellwood et al., 2005). This suggests that other photosynthetic organisms, as well as other ligand sources, such as the release of organic compounds during phytoplankton cell degradation, should be also considered.

The chemical nature of the ligands is not known, but it has been suggested that vitamin $\mathrm{B}_{12}$ and its degradation products can strongly bind Co (Saito et al., 2002, 2010), since Co is the structural atom of this vitamin. Siderophores, such as desferrioxamine B or siderophore-like compounds, can also form extremely strong complexes with Co (Duckworth et al., 2009). Cobalt may also be bound with humic substances in seawater (Yang and Van den Berg, 2009) although these complexes can be weaker than those formed with the vitamin $B_{12}$ and the coenzyme $\mathrm{B}_{12}$ (Ellwood and van den Berg, 2001), and with desferrioxamine B (Duckworth et al., 2009).

Here we report the distribution of Co-binding organic ligands in the water-column of the subtropical domain in the southeastern Atlantic and of the Antarctic Circumpolar Current and the Weddell Gyre in the Southern Ocean along the $0^{\circ}$ meridian to investigate their biogeochemical cycle in contrasted biogeochemical provinces and different watermasses. We use this information, along with the distribution of DCo recently described in the same area (Bown et al., 2011) to investigate the impact of organic speciation on the biogeochemical cycle of DCo in this region.

\section{Methods}

\subsection{Sampling}

Samples were collected during the multidisciplinary MD166 BONUS-GoodHope cruise that took place in the late austral summer 2008, during the International Polar Year (02/13/08 - 03/24/08) on board the French R/V Marion-Dufresne II sailing from Cape Town, South Africa, to $57^{\circ} \mathrm{S}$ along the Greenwich Meridian in the Southern Ocean (Fig. 1).

The samples for the determination of DCo and its organic speciation were collected from the upper $2100 \mathrm{~m}$ at 7 stations (referred as Large stations) and from the entire water column $(0-4000 \mathrm{~m})$ at 5 stations (referred as Super stations), with 10 acid-cleaned 12 L GO-FLO bottles (General Oceanics) modified with PTFE (polytetrafluoroethylene) rings, attached to a Kevlar wire and closed using Tefloncoated messengers. The samples were collected in acidcleaned LDPE (low-density polyethylene) Nalgene bottles after online filtration with $0.22 \mu \mathrm{m}$ Sartobran 300 (Sartorius) cartridge under pure $\mathrm{N}_{2}$ pressure $\left(99.99 \% \mathrm{~N}_{2}, 1\right.$ bar) in a pressurized clean container (class 100). Samples collected to assess the organic speciation of Co were immediately frozen at $-20^{\circ} \mathrm{C}$, and those collected for DCo analyses were acidified at $\mathrm{pH} \sim 2$ using ultrapure hydrochloric acid (ultrapurHCl, Merck) and stored in double bags in the dark at ambient temperature. The determination of the organic speciation of Co and the analyses of DCo were 


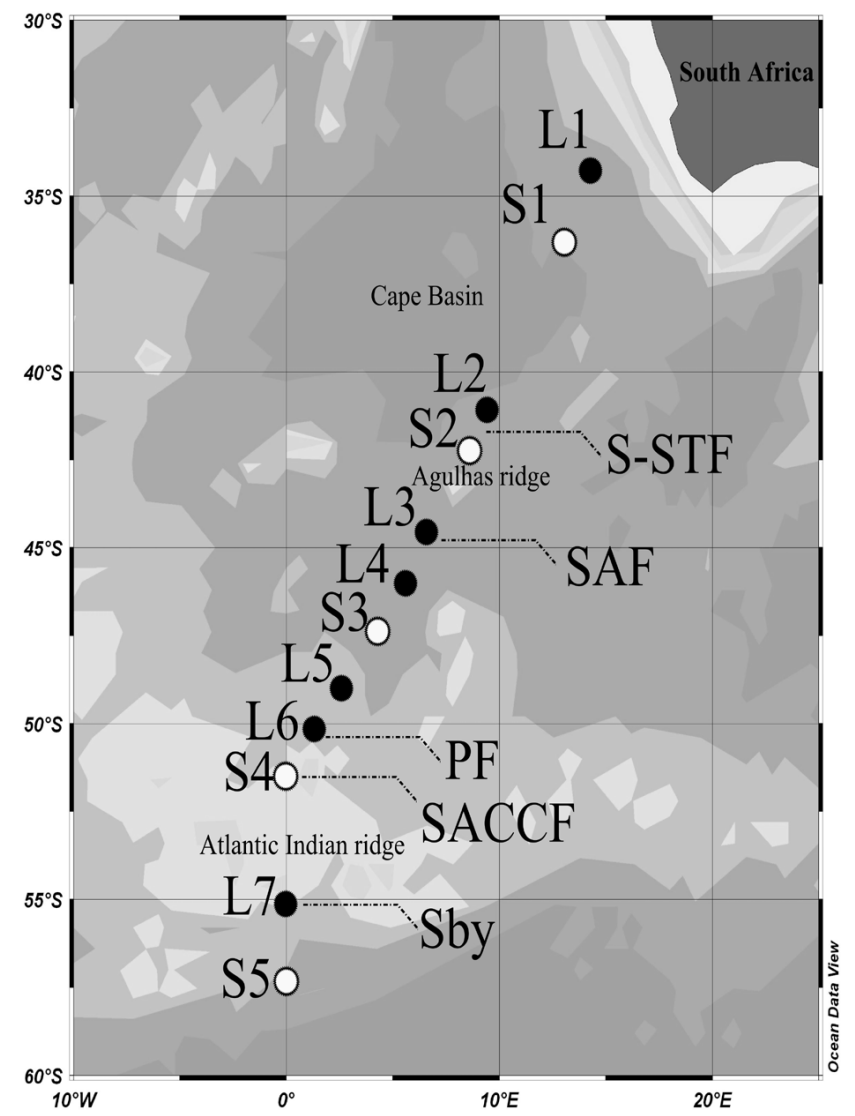

Fig. 1. Location of the stations sampled for dissolved cobalt (DCo) and its organic speciation during the MD166 BONUSGoodHope cruise. Black circles designate the LARGE stations and white circles the SUPER stations. The positions of fronts are also shown, with the southern branch of the subtropical front (S$\mathrm{STF} ; \sim 42^{\circ} 2^{\prime} \mathrm{S}$ ), the subAntarctic front (SAF; $44^{\circ} 2^{\prime} \mathrm{S}$ ), the polar front (PF; 50 $22.4^{\prime} \mathrm{S}$ ), the southern ACC front (SACCF; 51 ${ }^{\circ} 52^{\prime} \mathrm{S}$ ) and the southern boundary of the Antarctic Circumpolar Current (SBdy; $\sim 55^{\circ} 54.3^{\prime} \mathrm{S}$ ).

From their geographical positions, stations L1, S1 and L2 were in the subtropical domain (STD), S2 and L3 were, respectively, on the northern and southern side of the subantarctic zone (SAZ), stations L3, L4, S3 and L5 were within the polar frontal zone, station L6 was on the northern flank of PF, S4 was on the SACCF, station L7 was at the SBy, and station S5 was in the northern branch of the Weddell Gyre.

Figure prepared using Ocean Data View (Schlitzer, 2012).

conducted in a clean room in the shore-based laboratory, about 3 months and 18 months after the samples collection, respectively.

\subsection{Analytical procedures}

\section{Dissolved cobalt}

The concentrations of DCo were measured in acidified and UV-digested samples by flow injection analysis and chemi- luminescence detection as described in Bown et al. (2011). The reagent blank from acid/buffer additions and column rinse was $5.90 \pm 1.24 \mathrm{pM}$ (standard error, $n=40$ ) in Milli-Q grade water, yielding a detection limit $(3 \sigma)$ of $3.72 \mathrm{pM}(n=$ 40) (Bown et al., 2011).

\section{Organic speciation of dissolved cobalt}

The organic speciation of DCo was determined by complexing capacity titrations in the filtrate by competing ligand exchange-adsorptive cathodic stripping voltammetry (CLE-AdCSV) with ligand competition against nioxime at $\mathrm{pH} \sim 8.1$ (adapted from Zhang et al., 1990; Ellwood and van den Berg, 2001).

Two identical voltammetric devices, consisting of a mercury-drop electrode (model VA 663, Metrohm, Switzerland) connected to a $\mu$ Autolab potentiostat (Type II, Ecochemie) and an autosampler (Sample Processor 778, Metrohm) were used simultaneously to increase the sample throughput. The reference electrode was double junction, $\mathrm{Ag} / \mathrm{AgCl}, 3 \mathrm{M} \mathrm{KCl}$, with a salt bridge filled with $3 \mathrm{M} \mathrm{KCl}$, and the counter electrode was a glassy carbon rod.

The seawater $\mathrm{pH}$ was buffered at 8.1 using borate buffer $\left(\mathrm{H}_{3} \mathrm{BO}_{3}\right.$, Sigma Ultra). The borate stock solution (1 M boric acid/ $0.3 \mathrm{M} \mathrm{NaOH}$, Merck) was cleaned by running through a Chelex-100 column and was used at a final concentration of $10 \mathrm{mM}$. A stock solution of $0.1 \mathrm{M}$ nioxime (1,2cyclohexanedione dioxime, Sigma Aldrich) was prepared in $0.2 \mathrm{M}$ sodium hydroxide (Merck). A final nioxime concentration of $0.1 \mathrm{mM}$ was used for the titrations. Samples were titrated with Co using a stock Co-solution of $10^{-8} \mathrm{M}$ (Spectrosol solution, Sigma Aldrich) prepared in $0.05 \%$ (v:v) ultrapure hydrochloric acid, which was added in 10 increments on the order of $10 \mathrm{pM}$ to $200 \mathrm{pM} \mathrm{Co}$. Any remaining sample was pipetted into a 12th vial and used before the first measurement to condition the voltammetric cell. The $30 \mathrm{~mL}$ Teflon vials containing the sample and the reagents (Co standard, borate buffer and nioxime solution) were left for equilibration for $12-15 \mathrm{~h}$, at room temperature in a laminar flow hood.

The twelve equilibrated solutions were sequentially (starting from the lowest cobalt addition) and automatically transferred to the voltammetric Teflon cell. Once the cell was emptied, $3 \mathrm{~mL}$ of the sample solution were pumped to rinse the system and $15 \mathrm{~mL}$ then used for analysis. At the end of each titration, the voltammetric cell was rinsed with acidified MilliQ ( $\mathrm{HCl}$ ultrapure, $1^{0} \% \mathrm{v}: \mathrm{v}$ ), and thereafter with MilliQ water.

After purging each solution with $\mathrm{O}_{2}$-free nitrogen $(99.999 \%)$ for $3.25 \mathrm{~min}$, the measurements were made using a deposition time onto the mercury drop of $180 \mathrm{~s}$ at an adsorption potential of $-0.7 \mathrm{~V}$, followed by a $10 \mathrm{~s}$ equilibration period. Then the potential was scanned from -0.7 to $-1.2 \mathrm{~V}$ using the differential pulse waveform with a pulse amplitude of $100 \mathrm{mV}$, a modulation time of 
$10 \mathrm{~ms}$, an interval time of $100 \mathrm{~ms}$ and a step potential of $1.5 \mathrm{mV}$. Repeated (3 times) scan rates were made for each measurement, and the mean peak height of the reduction current of the Co-nioxime complex was used to resolve the titration.

The conditional stability constant for the Co-ligand complexes $(\mathrm{CoL})$ was defined as:

$\mathrm{K}^{\prime} \mathrm{CoL}=[\mathrm{CoL}] /\left(\left[\mathrm{Co}^{2+}\right] *\left[\mathrm{~L}^{\prime}\right]\right)$

where $[\mathrm{CoL}]$ is the organic Co concentration, $\left[\mathrm{Co}^{2+}\right]$ is the free Co ion concentration and [L'] is the concentration of organic ligands not bound to Co. The ligand concentration ([L]) is equal to the addition of the ligand concentrations bound to Co $([\mathrm{CoL}])$ to those not bound ([L']). $[\mathrm{L}]$ and $\mathrm{K}^{\prime} \mathrm{CoL}$ were calculated by linear least-squares regression of the data fitted to the Ruzic/van den Berg following equation (Ruzic, 1982; van den Berg, 1982; van den Berg and Kramer, 1979), from the slope and the Y-intercept respectively:

$$
\begin{aligned}
& {\left[\mathrm{Co}_{\text {labile }}\right] /[\mathrm{CoL}]=\left[\mathrm{Co}_{\text {labile }}\right] /[\mathrm{L}]} \\
& +\left(\alpha_{\mathrm{Co}}+\alpha_{\mathrm{CoNioxime} 2}\right) /\left([\mathrm{L}] * \mathrm{~K}^{\prime} \mathrm{CoL}\right)
\end{aligned}
$$

The inorganic side reaction coefficient for Co was taken as $\log \alpha_{\mathrm{Co}^{\prime}}=1.74$ (Turner et al., 1981), and for the $\mathrm{Co}^{-N i o x i m e}{ }_{2}$ complexes as $\log \alpha{ }^{\prime}$ CoNioxime2 $=7.62$ (using $\log \beta{ }^{\prime}{ }{ }$ oNioxime $2=15.62$ which was calibrated at salinity 35 and $\mathrm{pH} 7.8$ by Zhang et al. (1990), and $\left[\right.$ Nioxime $\left.\left._{2}\right]=0.1 \mathrm{mM}\right)$. The analytical detection window $\left(\right.$ centred on $\alpha{ }^{\prime}$ CoNioxime $2=\beta^{\prime}{ }_{\text {CoNioxime } 2}\left[\right.$ CoNioxime ${ }^{2}$ ) was then estimated in the range of $10^{6.62}$ to $10^{8.62}$ for the detection of $\alpha_{\mathrm{CoL}}$ (equal to [CoL'] $\times \mathrm{K}^{\prime} \mathrm{CoL}$ ). A rough estimate of 16.18 for $\log \beta^{\prime}{ }_{\text {CoNioxime } 2}$ is obtained at the working $\mathrm{pH}$ 8.1, using the linear equation between 15.62 at pH 7.8 (Zhang et al., 1990) and 18.10 at pH 9.1 (Ellwood and van den Berg, 2001). Hence, the value of 15.62 that we used for $\log \beta$ ' CoNioxime 2 is thus actually lower than the value expected at $\mathrm{pH} 8.1$. The conditional stability constants $\mathrm{K}^{\prime} \mathrm{CoL}$ we calculated are thus slightly underestimated.

The concentration of labile $\mathrm{Co}\left[\mathrm{CO}_{\text {labile }}\right]$ in each aliquot was calculated from the triplicate measured peak height $\left(\mathrm{i}_{\mathrm{p}}\right)$ as:

$\left[\mathrm{Co}_{\text {labile }}\right]=i_{\mathrm{p}} / \mathrm{S}$

where $\mathrm{S}$ is the sensitivity calculated from the linear part of the titration and $\mathrm{i}_{\mathrm{p}}$ the peak height obtained at the reduction potential of the Co-Nioxime 2 complex.

The concentration of Co-L complexes that equilibrates with the added nioxime and Co was calculated in Eq. (2) from:

$[\mathrm{Co}-\mathrm{L}]=[\mathrm{DCo}]-\left[\mathrm{Co}_{\text {labile }}\right]$ where [DCo] is the dissolved Co concentration including the Co additions.

Then [L], K' ${ }^{\prime} \mathrm{Co}$ and their standard deviations (stdv) were calculated from the slope and the Y-axis intercept of the linear least-squares regression of $\left[\mathrm{Co}_{\text {labile }}\right] /[\mathrm{CoL}]$ as a function of $\left[\mathrm{Co}_{\text {labile }}\right]$ (Eq. 2) fitted for a single ligand. The reproducibility obtained from triplicate analyses of a filtered seawater sampled at $30 \mathrm{~m}$ depth of station S5 (Fig. 1) was better than $10 \%$ for the ligand concentration determinations (e.g., $38.9 \pm 3.47 \mathrm{pM}, n=3$ ), and better than $1 \%$ for the conditional stability constant (e.g., log value of $18.1 \pm 0.21, n=3$ ).

The free metal ion concentration $\left(\left[\mathrm{Co}^{2+}\right]\right)$ originally present in the seawater is calculated with the following quadratic equation:

$$
\begin{aligned}
& {\left[\mathrm{Co}^{2+}\right]^{2} * \alpha_{\mathrm{Co}} * K_{\mathrm{CoL}}^{\prime}+\left[\mathrm{Co}^{2+}\right]} \\
& *\left(\alpha_{\mathrm{Co}}+\mathrm{K}^{\prime}{ }_{\mathrm{Co}} *[\mathrm{~L}] \mathrm{K}^{\prime}{ }_{\mathrm{Co}} *[\mathrm{DCo}]\right)-[\mathrm{DCo}]=0
\end{aligned}
$$

Then the inorganic Co concentration ([Co']) originally present in the seawater is calculated from:

$$
\left[\mathrm{Co}^{\prime}\right]=\alpha_{\mathrm{Co}^{\prime}} *\left[\mathrm{Co}^{2+}\right]
$$

The concentration of the organic cobalt $([\mathrm{CoL}])$ in the seawater is obtained by difference from the DCo concentration and the inorganic metal concentrations as:

$[\mathrm{CoL}]=[\mathrm{DCo}]-\left[\mathrm{Co}^{\prime}\right]$

The fraction (\%) of Co occurring as organic species is calculated as $([\mathrm{CoL}] /[\mathrm{DCo}]) * 100$.

\section{Results}

\subsection{Hydrography}

The cruise track crossed the subtropical domain southwest of South Africa (stations L1, S1, L2), the frontal systems (Belkin and Gordon, 1996; Orsi et al., 1995) of the Antarctic Circumpolar Current (ACC; stations S2 to L7) and entered the Weddell Gyre (station S5), as shown in Fig. 1. The water-masses and circulation patterns of the study area are given in detail in Bown et al. (2011). The vertical sections of salinity, potential temperature and dissolved oxygen are given in Fig. 2 (S. Speich et al., personal communication, 2011), and a glossary is provided in Table 2 to report the main water-masses, their origin and associated characteristics, and depth ranges.

\subsection{Biogeochemical features of the studied area}

Biogeochemical features are described and detailed elsewhere (Le Moigne et al., 2012). Several biogeochemical provinces were crossed along the section, generally characterized by a southward increase of macronutrient concentrations, much of which occurred near the frontal boundaries. Briefly, the subtropical domain (stations L1 to S2) displayed low nitrate, phosphate and silicate concentrations, and 


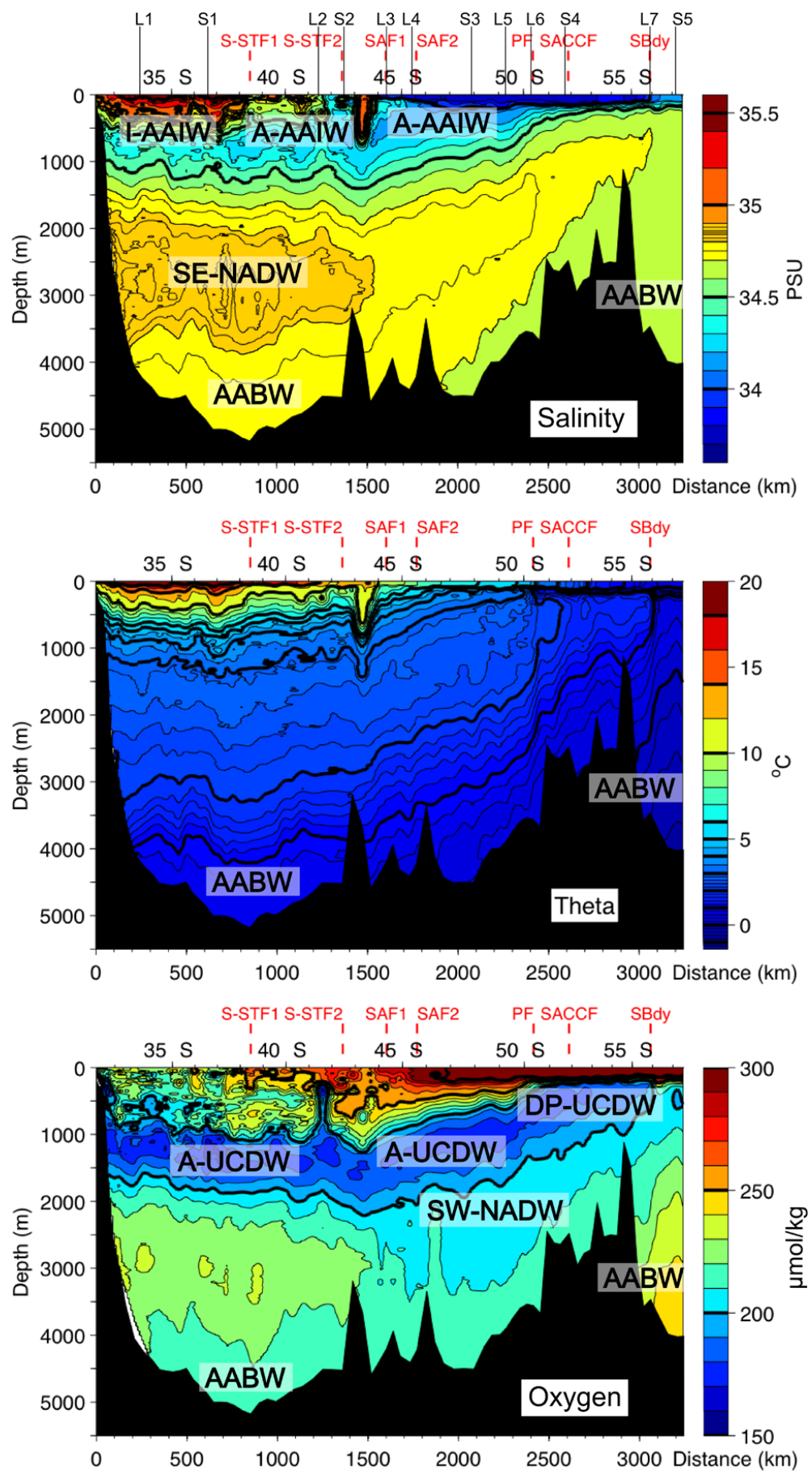

Fig. 2. Contour plots of salinity, potential temperature and oxygen versus depth along the MD166 BONUS-GoodHope transect from north (left) to the south (right) of the section (S. Speich et al., personal communication, 2011). The name, origin and characteristics of the watermasses are given in Table 2.

a subsurface Chl $a$ maximum with concentrations ranging between 0.3 and $0.6 \mu \mathrm{g} \mathrm{L}^{-1}$ (Fig. 3), characteristic of oligotrophic conditions (Le Moigne et al., 2012). In the polar frontal zone, near the PF, nitrate and phosphate concentrations increased while silicate remained low and ammonium exhibited a subsurface maximum of $\sim 1.25 \mu \mathrm{M}$ (Le Moigne et al., 2012). Chl $a$ concentrations ranged between 0.2 and $0.4 \mu \mathrm{g} \mathrm{L}^{-1}$, while phaeopigments, the degradation products of Chl $a$, displayed their highest values recorded along the section (Le Moigne et al., 2012). All these features indicated 
that a bloom of diatoms occurred before the start of our observations in the vicinity of the PF (Le Moigne et al., 2012). Macronutrient concentrations increased in the high-nutrient, low-chlorophyll area between stations S4 and L7 while Chl $a$ concentrations were low $\left(<0.3 \mu \mathrm{g} \mathrm{L}^{-1}\right.$; Fig. 3). A slight increase of Chl $a$ concentration up to $0.3 \mu \mathrm{g} \mathrm{L}^{-1}$ (Fig. 3), associated with an increase of phaeopigments and a slight decrease of silicate concentrations, was recorded at the southern end of the section at station S5, located in the Weddell Gyre, possibly indicating a recent, rather small bloom of diatoms (Le Moigne et al., 2012).

\subsection{Dissolved cobalt}

DCo concentrations ranged between $5.73 \pm 1.15 \mathrm{pM}$ and $72.9 \pm 4.51 \mathrm{pM}$ (standard deviations, based on triplicate analyses) along the section (Table 1; Bown et al., 2011). The distribution of DCo was nutrient-like in surface waters of the subtropical domain, with the lowest concentrations measured in the highest Chl $a$ maxima (Fig. 3; Bown et al., 2011). In deeper waters DCo distributions showed increasing concentrations below the nutricline, and higher concentrations in the intermediate (e.g.; I-AAIW) and deep (e.g.; SE-NADW) water-masses that have been in contact with the continental margins of South Africa (Bown et al., 2011). In the subantarctic region and beyond it (e.g. stations S2 to L4), DCo also still shows a nutrient-like profile with surface depletion in the euphotic layer and increasing concentration below $250 \mathrm{~m}$ (Fig. 3). In contrast, DCo was not depleted in the surface waters of the central and the southern ACC domains (Fig. 3) and exhibit lower concentrations in intermediate and deep waters (e.g. S3, L6; Fig. 3). Finally, the vertical distribution of DCo showed conservative distribution in the Weddell Gyre (Fig. 3; Bown et al., 2011). At depth, the highest DCo concentrations were observed in the cores of the low-oxygenated Upper Circumpolar Deep Waters (e.g. A-UCDW and DP-UCDW; Bown et al., 2011), and relatively high values were also observed in the core of A-AAIW; these waters originated from the southwest Atlantic and the Drake Passage. In the recently formed AABW near the Weddell Gyre seafloor, DCo concentrations were lower $(28.1 \pm 0.87 \mathrm{pM})$ than those measured in the older variety of AABW that flowed north of the Agulhas Ridge (40.0 \pm 2.49 pM; Bown et al., 2011).

\subsection{Organic Co-binding ligands}

The plots of $\left[\mathrm{Co}_{\text {labile }}\right] /[\mathrm{CoL}]$ versus $\left[\mathrm{Co}_{\text {labile }}\right]$ (e.g.; Eq. 2) were linear, indicating that the van den Berg/Ruzic equation fitted to 1:1 ligand:Co can be used to resolve the linear equation (Ruzic, 1982; van den Berg, 1982). The organic Co-binding ligand concentrations ([L]) ranged between $26.3 \pm 1.9 \mathrm{pM}$ (standard deviation of triplicate analyses) at the SACCF (at $1117 \mathrm{~m}$ depth at station S4) and 71.9 $\pm 2.8 \mathrm{pM}$ in the subtropical domain (STD) (at $15 \mathrm{~m}$ depth at sta- tion L2), as reported in Table 1, with a mean $\mathrm{L}$ value of $45.5 \pm 9.4 \mathrm{pM}(n=142)$ ( \pm corresponds to the standard deviation from the mean concentration unless otherwise stated).

The distribution of Co-binding ligands displayed relative [L] maxima in the Chl $a$ rich layer of the subtropical domain and northern subantarctic region $(\sim 50 \mathrm{pM}$ at stations L1, S1 and S2; $\sim 70$ pM at station L2; Fig. 3), as well as relative decreases (to 30 and $40 \mathrm{pM}$ ) at the depth of the DCo minimum (Fig. 3). High ligand concentrations (up to $72 \mathrm{pM}$ ) and the highest excess of ligands (mean value of $41.1 \pm 15.1 \mathrm{pM}, n=7$ ) over DCo concentrations (mean value of $12.8 \pm 5.68 \mathrm{pM}, n=7$ ) were observed in the upper $150 \mathrm{~m}$ at stations L2 and S2, both marked by a subsurface maximum in Chl- $a$ concentrations, with the highest Chl-a values recorded along the section concomitant with the lowest recorded DCo (Fig. 3). The highest $[\mathrm{L}] /[\mathrm{DCo}]$ ratio was also found in the surface waters at stations L2 and S2 with a mean of $3.90 \pm 2.10 \mathrm{pM} \mathrm{pM}^{-1}$ (Figs. 4-5), while the overall mean $[\mathrm{L}] /[\mathrm{DCo}]$ ratio was $1.22 \pm 0.78 \mathrm{pM} \mathrm{pM}^{-1} \quad(n=142)$. Below the nutricline, $[\mathrm{L}]$ increased to values similar or slightly higher than those in near surface waters (Fig. 3). In intermediate and deep waters, $\mathrm{L}$ distributions closely followed those of DCo and were in similar concentration ranges (Table 1, Fig. 3). Ligand concentrations were higher in the cores of I-AAIW, A-AAIW (e.g.; $49.4 \pm 2.4$ pM, $n=6$; and $50.8 \pm 11.1 \mathrm{pM}, n=5$, respectively), and in the core of SENADW (51.0 $\pm 3.9 \mathrm{pM}, n=7)$ than in the AABW (44.9 \pm 1.9 , $n=3)$.

Southward of the central ACC, the vertical distribution of $\mathrm{L}$ was fairly conservative in the upper $150 \mathrm{~m}$ with a mean [L] of $44.0 \pm 8.7 \mathrm{pM}(n=22)$ (Fig. 3, Table 1). In these surface waters the mean ligand concentration did not significantly exceed DCo levels (mean value $42.5 \pm 7.23 \mathrm{pM}, n=21$ ); this is also reflected by a fairly invariant $[\mathrm{L}] /[\mathrm{DCo}]$ ratio of $\sim 1 \mathrm{pM} \mathrm{pM}^{-1}$ (Figs. 4-5). In intermediate and deep waters, the concentrations and distributions of $\mathrm{L}$ were also similar to those of DCo (Fig. 3), with relatively high values of [L] recorded in the core of A-AAIW $(54.5 \pm 9.16 \mathrm{pM}, n=6)$, in A-UCDW (46.7 $\pm 9.02 \mathrm{pM}, n=4)$ and in DP-UCDW (46.5 \pm $9.15 \mathrm{pM}, n=10)$. In the AABW [L] were lower than in intermediate and deep waters and decreased southward from $39.2 \pm 1.74 \mathrm{pM}(n=2)$ at $\mathrm{S} 3$ to $32.7 \pm 0.78 \mathrm{pM}(n=2)$ at the southernmost station S5.

The overall mean conditional stability constant $\left(\log \mathrm{K}^{\prime} \mathrm{CoL}\right)$ was $18.8 \pm 0.38(n=142)$, and was not significantly different in the upper $150 \mathrm{~m}(18.7 \pm 0.46, n=41)$ and intermediate and deep waters $(18.9 \pm 0.33, n=101)$. The standard deviation of the conditional stability constants varied between (log values) 0.05 and 0.6 ; the highest values being reported in the samples where the initial DCo concentration was close or greater than the ligand concentration (Table 1). This bias is due to the absence of curvature of the titration which causes the intercept with the Y-axis (Eq. 2) to be small and the error to be relatively large. 
Table 1. All data of the organic speciation of dissolved cobalt obtained in the southeastern Atlantic and the Southern Ocean during the MD166 BONUS-GoodHope cruise in 2008: dissolved cobalt concentrations ([DCo]; Bown et al., 2011), the organic cobalt-binding ligand concentrations ([L]), the conditional stability constant of CoL complexes $\left(\mathrm{K}_{\mathrm{CoL}}\right)$, organic $(\mathrm{CoL})$ and inorganic $\left(\mathrm{Co}^{\prime}\right)$ cobalt concentrations, and the percentage of organically bound dissolved cobalt.

\begin{tabular}{|c|c|c|c|c|c|c|c|c|c|c|c|c|}
\hline Domain & Station & $\begin{array}{r}\text { Latitude } \\
\left({ }^{\circ} \mathrm{S}\right)\end{array}$ & $\begin{array}{r}\text { Longitude } \\
\left({ }^{\circ} \mathrm{E}\right)\end{array}$ & $\begin{array}{r}\text { Depth } \\
(\mathrm{m})\end{array}$ & $\begin{array}{r}\text { DCo } \\
(\mathrm{pM})\end{array}$ & $\begin{array}{r}\text { Stdv DCo } \\
(\mathrm{pM})\end{array}$ & $\begin{array}{r}\mathrm{L} \\
(\mathrm{pM})\end{array}$ & $\begin{array}{r}\text { Stdv L } \\
(\mathrm{pM})\end{array}$ & $\log \mathrm{K}^{\prime} \mathrm{CoL}$ & $\begin{array}{r}\mathrm{CoL} \\
(\mathrm{pM})\end{array}$ & $\begin{array}{r}(-) \log \mathrm{Co}^{\prime} \\
(\mathrm{pM})\end{array}$ & $\begin{array}{r}\text { Organic } \\
\text { cobalt }(\%)\end{array}$ \\
\hline \multirow[t]{10}{*}{ STZ } & L1 & 34.43 & 14.4 & 20 & 27.1 & 2.58 & 34.9 & 1.40 & 18.9 & 27.1 & 18.1 & $>99.9$ \\
\hline & & & & 40 & 29.7 & 2.55 & 35.1 & 0.80 & 18.8 & 29.7 & 17.8 & $>99.9$ \\
\hline & & & & 60 & 30.4 & 1.54 & 48.0 & 1.50 & 18.7 & 30.4 & 18.2 & $>99.9$ \\
\hline & & & & 80 & 29.9 & 0.67 & 32.7 & 1.90 & 18.5 & 29.9 & 17.2 & $>99.9$ \\
\hline & & & & 200 & 35.0 & 2.70 & 44.6 & 1.76 & 18.7 & 35.0 & 17.9 & $>99.9$ \\
\hline & & & & 700 & 50.1 & 3.09 & 39.3 & 1.00 & 19.3 & 39.3 & 11.0 & 78.4 \\
\hline & & & & 800 & 48.6 & 2.20 & 53.9 & 1.10 & 19.1 & 48.6 & 17.9 & $>99.9$ \\
\hline & & & & 1000 & 47.5 & 2.39 & 50.2 & 1.40 & 19.0 & 47.5 & 17.5 & $>99.9$ \\
\hline & & & & 1200 & 54.4 & 2.55 & 47.3 & 0.70 & 19.0 & 47.3 & 11.1 & 86.8 \\
\hline & & & & 2100 & 46.1 & 0.20 & 55.4 & 1.85 & 18.4 & 46.1 & 17.5 & $>99.9$ \\
\hline \multirow[t]{20}{*}{ STZ } & S1 & 36.5 & 13.1 & 20 & 23.7 & 2.15 & 47.8 & 0.67 & 16.6 & 23.7 & 16.4 & $>99.9$ \\
\hline & & & & 30 & 15.6 & 1.68 & 35.8 & 1.00 & 18.9 & 15.6 & 18.8 & $>99.9$ \\
\hline & & & & 40 & 26.3 & 1.97 & 34.9 & 1.00 & 19.2 & 26.3 & 15.5 & $>99.9$ \\
\hline & & & & 70 & 41.0 & 0.23 & 45.5 & 1.00 & 19.1 & 41.0 & 17.9 & $>99.9$ \\
\hline & & & & 100 & 44.3 & 0.73 & 45.2 & 2.00 & 19.2 & 44.3 & 17.2 & $>99.9$ \\
\hline & & & & 200 & 30.5 & 1.43 & 46.1 & 1.00 & 19.3 & 30.5 & 18.7 & $>99.9$ \\
\hline & & & & 300 & 37.6 & 2.80 & 44.3 & 2.00 & 19.1 & 37.6 & 18.1 & $>99.9$ \\
\hline & & & & 500 & 42.7 & 0.65 & 34.0 & 1.00 & 18.7 & 34.0 & 11.1 & 79.7 \\
\hline & & & & 700 & 51.8 & 0.53 & 52.5 & 1.00 & 19.1 & 51.8 & 16.9 & $>99.9$ \\
\hline & & & & 850 & 46.6 & 2.35 & 48.0 & 1.00 & 18.4 & 46.6 & 16.6 & $>99.9$ \\
\hline & & & & 1000 & 48.6 & 2.39 & 47.9 & 2.00 & 19.1 & 47.9 & 12.1 & 98.4 \\
\hline & & & & 1200 & 48.7 & 1.52 & 49.0 & 1.00 & 19.0 & 48.8 & 16.5 & $>99.9$ \\
\hline & & & & 1400 & 45.2 & 4.64 & 39.4 & 1.00 & 18.8 & 39.4 & 11.2 & 87.1 \\
\hline & & & & 1600 & 46.6 & 5.17 & 46.0 & 1.00 & 19.0 & 46.0 & 12.2 & 98.8 \\
\hline & & & & 2000 & 48.9 & 2.14 & 50.7 & 2.00 & 18.9 & 48.9 & 17.2 & $>99.9$ \\
\hline & & & & 2700 & 45.4 & 1.35 & 42.9 & 2.00 & 19.2 & 42.9 & 11.6 & 94.5 \\
\hline & & & & 3050 & 53.9 & 3.30 & 52.9 & 1.00 & 19.0 & 52.9 & 12.0 & 98.1 \\
\hline & & & & 3500 & 37.8 & 1.53 & 42.8 & 2.00 & 18.9 & 37.8 & 17.8 & $>99.9$ \\
\hline & & & & 3800 & 38.7 & 2.58 & 46.5 & 2.00 & 19.1 & 38.7 & 18.2 & $>99.9$ \\
\hline & & & & 4000 & 41.3 & 2.39 & 45.4 & 1.00 & 19.1 & 45.4 & 11.5 & 93.4 \\
\hline \multirow[t]{10}{*}{ STZ } & L2 & 41.18 & 9.92 & 15 & 17.0 & 0.54 & 71.9 & 2.80 & 18.5 & 17.0 & 18.8 & $>99.9$ \\
\hline & & & & 35 & 15.3 & 3.19 & 35.0 & 1.13 & 18.8 & 15.3 & 18.7 & $>99.9$ \\
\hline & & & & 45 & 19.0 & 0.23 & 35.5 & 0.77 & 18.8 & 19.0 & 18.5 & $>99.9$ \\
\hline & & & & 95 & 17.7 & 1.08 & 26.4 & 0.99 & 19.0 & 17.7 & 18.5 & $>99.9$ \\
\hline & & & & 300 & 46.6 & 5.63 & 48.7 & 1.31 & 19.1 & 46.6 & 17.5 & $>99.9$ \\
\hline & & & & 600 & 54.6 & 4.30 & 62.6 & 2.10 & 18.8 & 54.6 & 17.7 & $>99.9$ \\
\hline & & & & 800 & 54.0 & 3.33 & 49.4 & 1.24 & 19.7 & 49.4 & 11.3 & 91.5 \\
\hline & & & & 1200 & 46.3 & 0.95 & 39.0 & 1.18 & 19.7 & 39.0 & 11.1 & 84.2 \\
\hline & & & & 1400 & 45.9 & 1.44 & 47.3 & 0.72 & 19.2 & 45.9 & 17.4 & $>99.9$ \\
\hline & & & & 2100 & 49.2 & 2.38 & 52.7 & 0.64 & 20.3 & 49.2 & 18.9 & $>99.9$ \\
\hline \multirow[t]{15}{*}{ SAZ } & $\mathrm{S} 2$ & 42.47 & 8.93 & 15 & 7.40 & 0.67 & 49.0 & 3.85 & 18.5 & 7.40 & 19.0 & $>99.9$ \\
\hline & & & & 30 & 5.73 & 1.15 & 35.6 & 0.80 & 19.0 & 5.73 & 19.5 & $>99.9$ \\
\hline & & & & 35 & 7.50 & 1.39 & 34.4 & 3.10 & 18.4 & 7.50 & 18.8 & $>99.9$ \\
\hline & & & & 196 & 38.2 & 0.99 & 69.2 & 5.80 & 17.9 & 38.2 & 17.5 & $>99.9$ \\
\hline & & & & 314 & 44.2 & 1.14 & 49.5 & 3.92 & 18.3 & 44.2 & 17.1 & $>99.9$ \\
\hline & & & & 461 & 52.1 & 2.66 & 32.8 & 2.68 & 18.5 & 32.8 & 10.7 & 62.8 \\
\hline & & & & 809 & 49.7 & 2.21 & 40.5 & 2.74 & 18.6 & 40.5 & 11.0 & 81.5 \\
\hline & & & & 1029 & 41.0 & 1.30 & 37.1 & 2.74 & 18.8 & 37.1 & 11.4 & 90.5 \\
\hline & & & & 1250 & 59.6 & 6.54 & 49.2 & 1.96 & 18.8 & 49.2 & 11.0 & 82.7 \\
\hline & & & & 1441 & 65.6 & 0.86 & 69.7 & 2.10 & 19.0 & 65.6 & 17.6 & $>99.9$ \\
\hline & & & & 1764 & 43.4 & 1.84 & 46.0 & 5.79 & 18.2 & 43.4 & 16.8 & $>99.9$ \\
\hline & & & & 2156 & 45.1 & 1.79 & 50.4 & 1.50 & 18.5 & 45.1 & 17.3 & $>99.9$ \\
\hline & & & & 2548 & 59.2 & 2.05 & 52.1 & 2.50 & 18.8 & 52.1 & 11.1 & 88.0 \\
\hline & & & & 3245 & 41.8 & 4.14 & 53.0 & 3.22 & 18.7 & 41.8 & 17.9 & $>99.9$ \\
\hline & & & & 3636 & 40.1 & 1.65 & 49.9 & 2.32 & 19.3 & 40.1 & 18.5 & $>99.9$ \\
\hline \multirow[t]{9}{*}{ SAZ } & L3 & 44.88 & 6.88 & 30 & 22.8 & 1.34 & 37.7 & 0.90 & 18.8 & 22.8 & 18.4 & $>99.9$ \\
\hline & & & & 100 & 38.7 & 0.83 & 46.7 & 1.60 & 19.3 & 38.7 & 18.3 & $>99.9$ \\
\hline & & & & 150 & 50.7 & 1.53 & 62.3 & 0.90 & 19.4 & 50.8 & 18.5 & $>99.9$ \\
\hline & & & & 270 & 49.3 & 2.50 & 58.4 & 2.20 & 19.0 & 49.4 & 18.0 & $>99.9$ \\
\hline & & & & 400 & 54.9 & 9.41 & 66.4 & 1.30 & 18.9 & 55.0 & 17.9 & $>99.9$ \\
\hline & & & & 600 & 47.2 & 3.56 & 53.8 & 2.70 & 18.8 & 47.2 & 17.7 & $>99.9$ \\
\hline & & & & 1200 & 49.5 & 1.04 & 55.7 & 1.40 & 19.2 & 49.5 & 18.1 & $>99.9$ \\
\hline & & & & 1400 & 51.9 & 1.21 & 51.6 & 1.60 & 18.9 & 51.6 & 12.5 & 99.4 \\
\hline & & & & 2100 & 47.1 & 1.01 & 41.0 & 1.10 & 19.2 & 41.0 & 11.2 & 87.1 \\
\hline
\end{tabular}


Table 1. Continued.

\begin{tabular}{|c|c|c|c|c|c|c|c|c|c|c|c|c|}
\hline Domain & Station & $\begin{array}{r}\text { Latitude } \\
\left({ }^{\circ} \mathrm{S}\right)\end{array}$ & $\begin{array}{r}\text { Longitude } \\
\left({ }^{\circ} \mathrm{E}\right)\end{array}$ & $\begin{array}{r}\text { Depth } \\
(\mathrm{m})\end{array}$ & $\begin{array}{l}\text { DCo } \\
\text { (pM) }\end{array}$ & $\begin{array}{r}\text { Stdv DCo } \\
(\mathrm{pM})\end{array}$ & $\begin{array}{r}\mathrm{L} \\
(\mathrm{pM})\end{array}$ & $\begin{array}{r}\text { Stdv L } \\
(\mathrm{pM})\end{array}$ & $\log \mathrm{K}^{\prime} \mathrm{CoL}$ & $\begin{array}{l}\mathrm{CoL} \\
(\mathrm{pM})\end{array}$ & $\begin{array}{r}(-) \log C o \\
(\mathrm{pM})\end{array}$ & $\begin{array}{r}\text { Organic } \\
\text { cobalt }(\%)\end{array}$ \\
\hline \multirow[t]{10}{*}{ SAZ } & \multirow[t]{10}{*}{$\mathrm{L} 4$} & \multirow[t]{10}{*}{46.01} & 5.87 & 30 & 27.3 & $x$ & 44.9 & 3.60 & 18.2 & 27.3 & 17.7 & $>99.9$ \\
\hline & & & & 60 & 29.1 & 1.66 & 28.5 & 1.30 & 18.7 & 28.5 & 12.2 & 97.9 \\
\hline & & & & 100 & 48.2 & 1.90 & 29.5 & 1.70 & 18.5 & 29.5 & 10.7 & 61.2 \\
\hline & & & & 150 & 40.5 & 1.86 & 46.4 & 2.60 & 18.6 & 40.5 & 17.5 & $>99.9$ \\
\hline & & & & 270 & 52.9 & 1.28 & 46.0 & 1.30 & 18.8 & 46.0 & 11.2 & 87.0 \\
\hline & & & & 400 & 49.2 & 2.01 & 44.0 & 1.40 & 18.9 & 44.0 & 11.3 & 89.4 \\
\hline & & & & 800 & 48.7 & 2.12 & 39.9 & 1.70 & 19.0 & 39.9 & 11.1 & 81.9 \\
\hline & & & & 1300 & 45.9 & 2.32 & 44.5 & 1.80 & 18.6 & 44.5 & 11.9 & 97.0 \\
\hline & & & & 1600 & 50.3 & 0.50 & 38.5 & 1.20 & 19.0 & 38.5 & 10.9 & 76.6 \\
\hline & & & & 2050 & 45.5 & 2.53 & 32.0 & 2.20 & 18.8 & 32.0 & 10.9 & 70.4 \\
\hline PFZ & S3 & 47.55 & 4.37 & 30 & 33.6 & 0.85 & 37.3 & 0.60 & 18.8 & 33.6 & 17.60 & $>99.9$ \\
\hline & & & & 70 & 35.7 & 1.27 & 44.4 & 3.00 & 18.3 & 35.7 & 17.40 & $>99.9$ \\
\hline & & & & 100 & 42.9 & 1.80 & 37.2 & 2.20 & 18.8 & 37.2 & 11.20 & 86.7 \\
\hline & & & & 200 & 40.5 & 2.17 & 37.8 & 1.50 & 18.8 & 37.8 & 11.60 & 93.4 \\
\hline & & & & 300 & 63.1 & 3.50 & 64.8 & 1.60 & 18.7 & 63.1 & 16.90 & $>99.9$ \\
\hline & & & & 450 & 50.1 & 0.30 & 49.8 & 2.40 & 18.4 & 49.8 & 12.50 & 99.4 \\
\hline & & & & 1500 & 42.3 & 0.28 & 35.1 & 1.60 & 19.2 & 35.1 & 11.10 & 83.0 \\
\hline & & & & 2020 & 39.5 & 1.84 & 43.6 & 1.60 & 18.9 & 39.5 & 17.60 & $>99.9$ \\
\hline & & & & 2500 & 40.7 & 3.22 & 49.2 & 1.10 & 19.2 & 40.7 & 18.30 & $>99.9$ \\
\hline & & & & 3000 & 36.6 & 0.91 & 63.4 & 2.00 & 19.1 & 36.6 & 18.70 & $>99.9$ \\
\hline & & & & 3500 & 33.3 & 3.20 & 40.5 & 0.80 & 18.9 & 33.3 & 18.00 & $>99.9$ \\
\hline & & & & 3980 & 30.5 & 0.27 & 38.0 & 1.20 & 19.1 & 30.5 & 18.30 & $>99.9$ \\
\hline PFZ & L5 & 49.03 & 2.84 & 40 & 43.5 & 2.33 & 35.5 & 2.80 & 18.2 & 35.5 & 11.10 & 81.6 \\
\hline & & & & 80 & 43.8 & 2.93 & 41.0 & 1.70 & 18.7 & 41.0 & 11.50 & 93.5 \\
\hline & & & & 150 & 42.2 & 3.23 & 44.3 & 1.10 & 19.2 & 42.3 & 17.60 & $>99.9$ \\
\hline & & & & 170 & 50.2 & 1.56 & 43.8 & 1.30 & 18.6 & 43.8 & 11.20 & 87.2 \\
\hline & & & & 250 & 49.0 & 0.95 & 39.8 & 1.70 & 18.6 & 39.8 & 11.00 & 81.2 \\
\hline & & & & 350 & 53.9 & 2.25 & 48.3 & 2.10 & 18.7 & 48.3 & 11.30 & 89.6 \\
\hline & & & & 700 & 72.9 & 4.51 & 63.6 & 2.60 & 18.8 & 63.6 & 11.00 & 87.2 \\
\hline & & & & 1600 & 46.0 & 1.10 & 50.8 & 1.60 & 18.9 & 46.0 & 17.70 & $>99.9$ \\
\hline & & & & 2200 & 39.9 & 1.26 & 47.7 & 2.60 & 19.1 & 39.9 & 18.10 & $>99.9$ \\
\hline North PF & L6 & 50.38 & 1.33 & 30 & 39.0 & 1.54 & 42.8 & 2.20 & 18.9 & 39.0 & 17.70 & $>99.9$ \\
\hline & & & & 60 & 37.8 & 3.27 & 38.6 & 2.40 & 19.0 & 37.8 & 17.10 & $>99.9$ \\
\hline & & & & 100 & 51.0 & 1.52 & 46.2 & 1.40 & 18.8 & 46.2 & 11.30 & 90.6 \\
\hline & & & & 135 & 58.7 & 2.19 & 60.5 & 2.00 & 18.9 & 58.7 & 17.20 & $>99.9$ \\
\hline & & & & 180 & 52.0 & 1.72 & 52.0 & 1.20 & 19.1 & 52.0 & 13.40 & $>99.9$ \\
\hline & & & & 300 & 48.6 & 0.46 & 47.5 & 1.90 & 19.2 & 47.5 & 12.00 & 97.8 \\
\hline & & & & 600 & 48.0 & 1.97 & 47.1 & 1.80 & 18.9 & 47.1 & 12.00 & 98.1 \\
\hline & & & & 850 & 45.1 & 3.06 & 48.9 & 1.30 & 18.9 & 45.1 & 17.60 & $>99.9$ \\
\hline & & & & 1600 & 39.5 & 2.22 & 49.6 & 1.10 & 19.1 & 39.5 & 18.20 & $>99.9$ \\
\hline & & & & 2100 & 36.5 & 1.74 & 44.7 & 1.70 & 18.9 & 36.5 & 18.00 & $>99.9$ \\
\hline South PF & $\mathrm{S} 4$ & 51.85 & 0 & 30 & 40.3 & 0.87 & 43.6 & 4.30 & 18.1 & 40.3 & 16.80 & $>99.9$ \\
\hline & & & & 60 & 37.6 & 2.10 & 35.0 & 2.20 & 18.2 & 35.0 & 11.60 & 93.3 \\
\hline & & & & 130 & 48.8 & 1.09 & 44.8 & 4.50 & 18.4 & 44.8 & 11.40 & 91.7 \\
\hline & & & & 160 & 45.6 & 2.55 & 52.1 & 1.10 & 19.0 & 45.6 & 17.90 & $>99.9$ \\
\hline & & & & 180 & 50.4 & 2.04 & 45.0 & 2.40 & 18.6 & 45.0 & 11.30 & 89.2 \\
\hline & & & & 250 & 56.8 & 1.40 & 49.7 & 2.50 & 18.8 & 49.7 & 11.10 & 87.4 \\
\hline & & & & 300 & 55.2 & 2.27 & 61.6 & 2.80 & 19.0 & 55.2 & 17.80 & $>99.9$ \\
\hline & & & & 350 & 49.6 & 1.33 & 49.2 & 1.80 & 18.9 & 49.2 & 12.40 & 99.2 \\
\hline & & & & 400 & 50.4 & 6.14 & 56.8 & 1.20 & 18.9 & 50.4 & 17.70 & $>99.9$ \\
\hline & & & & 500 & 50.1 & 1.66 & 42.1 & 0.80 & 18.9 & 42.1 & 11.10 & 84.0 \\
\hline & & & & 700 & 47.1 & 2.98 & 35.4 & 1.90 & 18.7 & 35.4 & 10.9 & 75.1 \\
\hline & & & & 1117 & 36.5 & 3.40 & 26.3 & 1.90 & 18.8 & 26.3 & 11.0 & 72.2 \\
\hline & & & & 1950 & 62.9 & 8.04 & 59.0 & 2.40 & 18.8 & 59.0 & 11.40 & 93.9 \\
\hline & & & & 2490 & 28.6 & 0.95 & 55.7 & 6.60 & 18.3 & 28.6 & 18.10 & $>99.9$ \\
\hline Sbdy & L7 & 55.23 & 0.03 & 30 & 51.3 & 1.11 & 52.0 & 1.80 & 19.1 & 51.3 & 17.00 & $>99.9$ \\
\hline & & & & 100 & 47.7 & 2.54 & 54.0 & 2.40 & 18.8 & 47.7 & 17.70 & $>99.9$ \\
\hline & & & & 120 & 49.7 & 1.88 & 49.4 & 2.60 & 18.7 & 49.4 & 12.50 & 99.3 \\
\hline & & & & 200 & 56.2 & 4.46 & 58.7 & 1.30 & 19.0 & 56.2 & 17.40 & $>99.9$ \\
\hline & & & & 300 & 49.5 & 3.15 & 56.0 & 1.80 & 19.1 & 49.5 & 18.00 & $>99.9$ \\
\hline & & & & 650 & 43.9 & 3.79 & 51.2 & 2.20 & 18.7 & 43.9 & 17.70 & $>99.9$ \\
\hline & & & & 1000 & 46.5 & 2.79 & 64.9 & 1.20 & 19.1 & 46.5 & 18.40 & $>99.9$ \\
\hline & & & & 1500 & 33.1 & 1.89 & 48.5 & 1.00 & 18.9 & 33.1 & 18.40 & $>99.9$ \\
\hline & & & & 2100 & 44.7 & 0.59 & 63.1 & 1.30 & 18.6 & 44.7 & 18.00 & $>99.9$ \\
\hline Weddell Gyre & S5 & 57.55 & 0.03 & 30 & 36.7 & 0.49 & 42.7 & 1.41 & 18.7 & 30.7 & 17.70 & $>99.9$ \\
\hline & & & & 60 & 36.0 & 0.60 & 49.8 & 4.50 & 18.2 & 22.2 & 17.5 & $>99.9$ \\
\hline & & & & 120 & 32.1 & 1.86 & 38.5 & 1.41 & 18.8 & 25.7 & 17.80 & $>99.9$ \\
\hline & & & & 250 & 43.4 & 1.23 & 38.5 & 2.53 & 18.9 & 43.4 & 11.30 & 89.0 \\
\hline & & & & 350 & 36.4 & 2.22 & 40.8 & 1.33 & 18.8 & 32.0 & 17.70 & $>99.9$ \\
\hline & & & & 450 & 39.7 & 2.74 & 35.7 & 2.50 & 18.6 & 39.7 & 11.40 & 90.0 \\
\hline & & & & 550 & 34.4 & 4.52 & 34.4 & 1.90 & 18.3 & 34.4 & 13.90 & $>99.9$ \\
\hline & & & & 800 & 37.6 & 4.03 & 37.2 & 0.80 & 18.6 & 37.6 & 12.40 & 99.0 \\
\hline & & & & 1250 & 30.1 & 1.91 & 35.2 & 1.88 & 18.7 & 25.0 & 17.70 & $>99.9$ \\
\hline & & & & 1700 & 38.4 & 0.65 & 43.7 & 0.75 & 19.2 & 33.1 & 18.10 & $>99.9$ \\
\hline & & & & 2150 & 32.9 & 1.26 & 36.1 & 2.21 & 18.5 & 29.8 & 17.30 & $>99.9$ \\
\hline & & & & 2600 & 33.3 & 3.01 & 44.0 & 1.28 & 18.7 & 22.6 & 18.0 & $>99.9$ \\
\hline & & & & 3500 & 29.1 & 1.01 & 32.1 & 1.10 & 18.9 & 26.2 & 17.60 & $>99.9$ \\
\hline & & & & 3840 & 30.5 & 0.32 & 33.2 & 1.26 & 18.7 & 27.7 & 17.50 & $>99.9$ \\
\hline
\end{tabular}




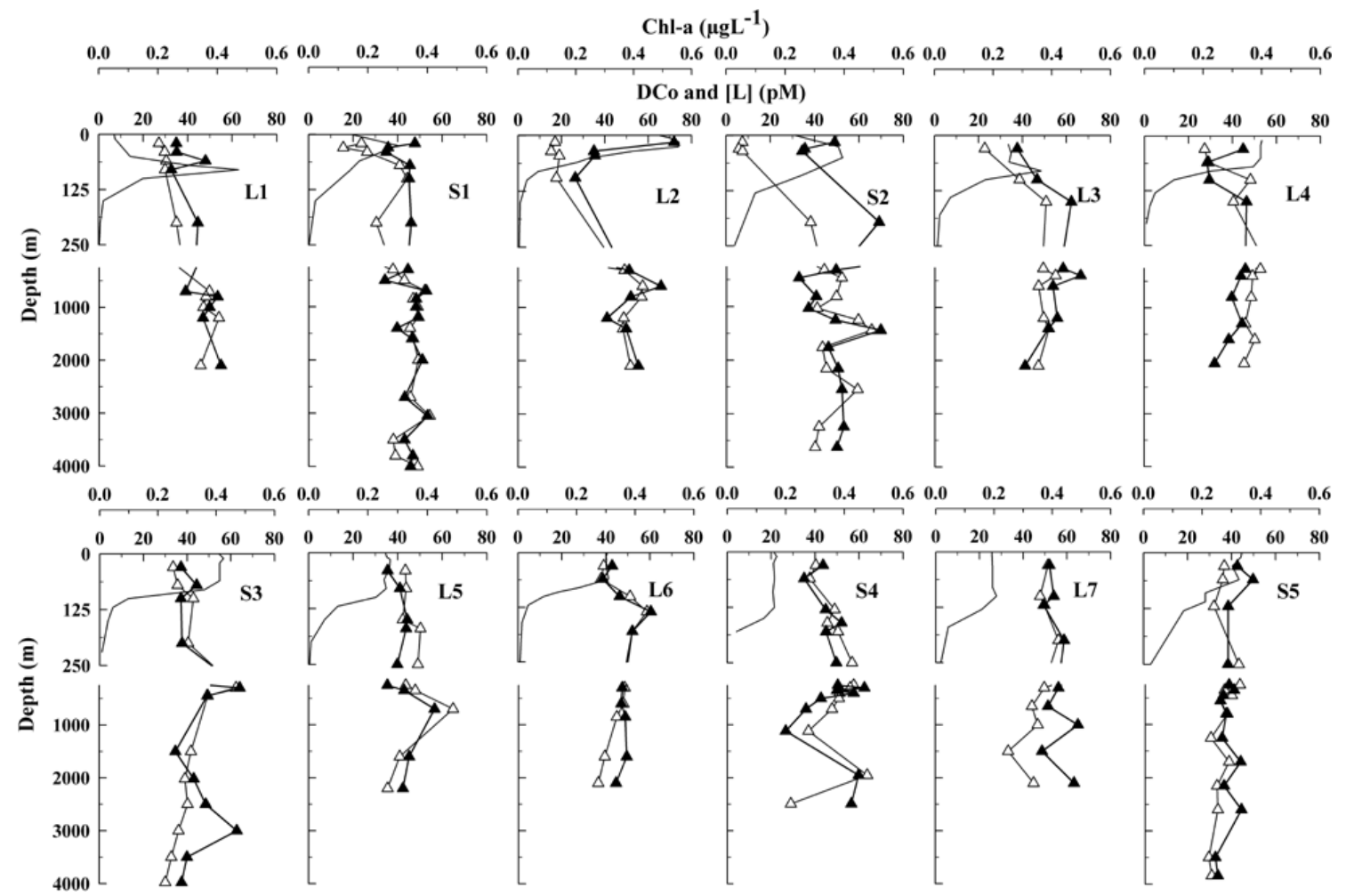

Fig. 3. Vertical distribution of the organic cobalt-binding ligands (L: black triangle), dissolved cobalt (DCo: white triangle; Bown et al., 2011), and Chlorophyll $a$ (Chl $a$ : solid line) at the LARGE and SUPER stations during the MD166 BONUS-GoodHope cruise. Chlorophyll $a$ data from Guéneuguès and Boye (2008).

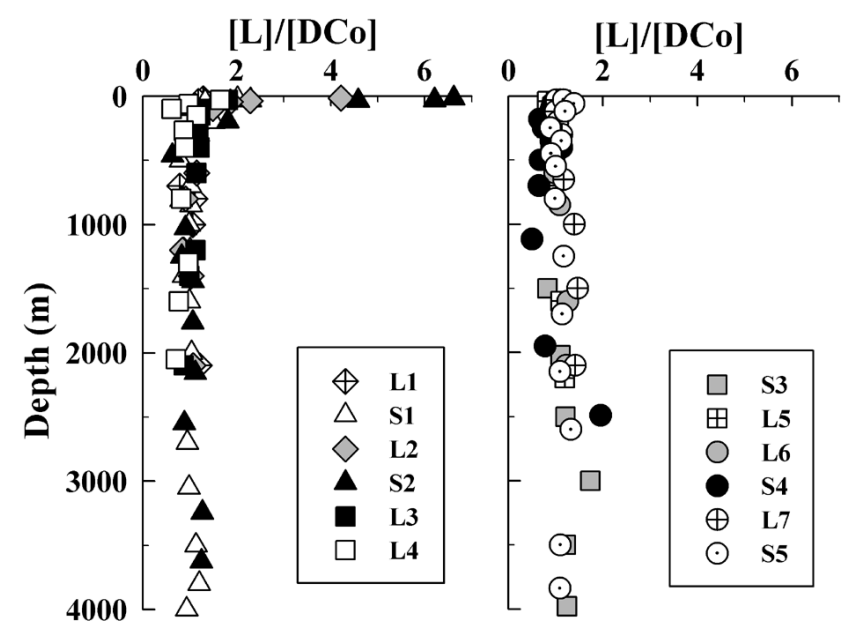

Fig. 4. Vertical distribution of L/DCo picomolar ratios in the subtropical and subantarctic domains (left panel), and in the polar frontal zone, the southern ACC and the northern Weddell Gyre (right panel).

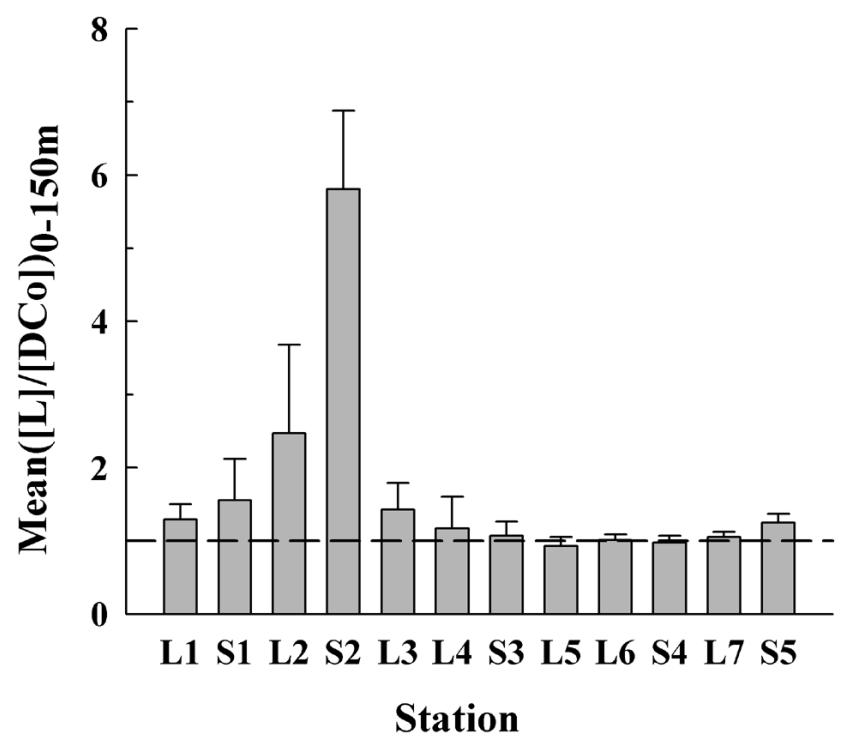

Fig. 5. Meridional trend of mean $[\mathrm{L}] /[\mathrm{DCo}]$ picomolar ratios averaged in the upper $150 \mathrm{~m}$ at stations $\mathrm{L}$ and $\mathrm{S}$ (the northern station is L1 on the left, the southern is S5 on the right). Dashed line represents mean $[\mathrm{L}] /[\mathrm{DCo}]$ of 1 . 


\subsection{Organic speciation of dissolved cobalt}

The organic complexation dominated the chemical speciation of DCo along the section, with DCo being organically bound at $\sim 60$ to $>99.9 \%$. In the surface waters of the subtropical and northern subantarctic domains, DCo was consistently $>99.9 \%$ organically bound (Table 1 ). Hence, inorganic Co concentrations ([Co']) were extremely low in these surface waters $\left(3 \times 10^{-20}-10^{-17} \mathrm{M}\right)$, whereas organic Co concentrations ([CoL]) were almost equal to [DCo], ranging between $\sim 5$ and $\sim 15 \mathrm{pM}$ (Table 1). At intermediate depths, in the cores of I-AAIW and A-AAIW, [Co'] was generally higher than in the surface layer with concentrations ranging from $\sim 1.0 \times 10^{-18}$ to $9.0 \times 10^{-12} \mathrm{M}$, and the organic Co represented $\sim 60$ to $>99.9 \%$ of DCo, lower than in the surface layer (Table 1). The deep SE-NADW was also characterized by higher inorganic Co levels $\left(4 \times 10^{-18}-7 \times 10^{-12} \mathrm{M}\right)$, with organic Co representing a lower fraction of DCo than in surface waters (although still 88 to $>99.9 \%$; Table 1). In the AABW, [Co'] varied between $6 \times 10^{-19}$ and $3 \times 10^{-12} \mathrm{M}$ and organic Co represented $\sim 93$ to $>99.9 \%$ of DCo. Southward of the central ACC the organic complexation still dominated the speciation of DCo, but its magnitude in surface waters was generally lower $(\sim 60$ to $>99.9 \%)$ than in the northern part of the section (Table 1). The higher DCo concentrations in the more southerly surface waters (Fig. 3) corresponded to higher inorganic Co concentrations, which could be as high as $11 \mathrm{pM}$ (Table 1), as well as higher organic Co concentrations (e.g.; 20-55 pM) compared to the northern surface waters (e.g.; 5-15 pM). In deeper waters the two varieties of UCDW were characterized by the organic Co representing $\sim 75$ to $>99.9 \%$ of DCo, and by Co' representing up to $25 \%$ ([Co'] $\sim 9 \times 10^{-19}$ and $\sim 8 \times 10^{-12} \mathrm{M}$, Table 1 ). In the AABW, [Co'] varied between $5-10 \times 10^{-19} \mathrm{M}$ (at station S3) and $3 \times 10^{-12} \mathrm{M}$ (at station S5) and the organic Co represented $90 \%$ (at station S5) to $>99 \%$ (at station S3) of DCo (Table 1).

\section{Discussion}

\subsection{Determination of cobalt speciation}

In most cases, the CSV peak height was found to increase linearly with Co additions, indicating that either relatively strong Co-binding organic ligands were already saturated with $\mathrm{Co}$, and/or that the nioxime was out competing the natural ligands for Co (Fig. 6a). The conditional stability constant of the ligand $\left(\mathrm{K}^{\prime} \mathrm{CoL}\right)$ cannot be calculated accurately in these conditions. The titration curves were however always below the theoretical curve that would be obtained if no ligand was present in the sample, or if the ligands were outcompeted for binding Co by the addition of nioxime. Hence there was in any case a larger fraction of detectable non-labile Co (e.g.; bound to strong ligands) with the labile fraction (e.g.; bound to nioxime) remaining far below the DCo concentration (Fig. 6a). Such saturation of the ligands with Co has already been reported in oceanic waters (Saito and Moffett, 2001; Zhang et al., 1990). In contrast, some titrations showed a curvature at low Co additions, indicative of the presence of free Co-binding sites (Fig. 6b).

The absence of curvature in most of the titrations is probably due to the presence of strong ligands saturated in $\mathrm{Co}(\mathrm{III})$, and to the rather low detection window (e.g.; centred on $\alpha^{\prime}$ CoNioxime $2=10^{7.62}$ ) which does not allow competitive equilibrium with these strong ligands $\left(\alpha_{\mathrm{CoL}}=10^{8.56 \pm 0.47}\right.$, $n=142$ ). The thermodynamically strong $\mathrm{Co}(\mathrm{III})-\mathrm{L}$ complexes, such as those formed with vitamin $\mathrm{B}_{12}$ (Menzel and Spaeth, 1962) or desferrioxamine B (Duckworth et al., 2009), can indeed outcompete the thermodynamically less stable Co(II)-nioxime complexes (Saito and Moffett, 2001). The organic complexes formed with $\mathrm{Co}(\mathrm{III})$ are stronger (e.g., $\log \mathrm{K}_{\mathrm{Co}(\mathrm{IIII}) \mathrm{EDTA}}=41.4$; (Xue and Traina, 1996)) than those formed with $\mathrm{Co}(\mathrm{II})$ (e.g., $\log \mathrm{K}_{\mathrm{Co}(\mathrm{II}) \mathrm{EDTA}}=16.45$; Martell and Smith, 1977). In these later cases, a second class of weak organic ligand (such as Co(II)-complexes, Saito and Moffett, 2001) is probably not involved, since the titrations could be solved with a $1 / 1$ model. On the other hand, it is also possible that any ligands in excess of Co were masked by the complexation with another metal ion, especially with nickel, which may be present at a much greater abundance than Co and has shown similar binding strength of organic complexes (Saito and Moffett, 2001).

\subsection{Distribution and cycle of the organic Co-binding ligands}

\section{Comparison with other studies}

Relatively few studies of Co-binding ligand distributions have been reported in the open ocean, but they provide ranges of ligand concentrations of the same general magnitude reported here (Fig. 7). The distributions of [L] recorded in the Sargasso Sea at station BATS (Bermuda Atlantic time-series study) show an overall excess of L over DCo in the euphotic layer associated with relative maxima of $[\mathrm{L}]$ in the chlorophyll maximum, while the difference between $\mathrm{L}$ and DCo decreased in deeper waters (Saito and Moffett, 2001); similar to the trends we observed in the subtropical and the subantarctic domains (Fig. 7). In the Atlantic sector of the Southern Ocean, [L] exceed [DCo] in surface waters south of the Polar Front $(\mathrm{PF})$, whereas the reverse was found to the north (Ellwood et al., 2005). Contrastingly, the difference between [L] and $[\mathrm{DCo}]$ remained almost constant across the Polar Front in our study, and was rather small north of the PF (Fig. 5). An overall excess of DCo over L was also observed in the surface waters of the North Atlantic (Ellwood and van den Berg, 2001; Fig. 7). These contrasted observations can reflect the spatial and seasonal variability of processes that drive $\mathrm{L}$ production and degradation. In waters below $150 \mathrm{~m}$, there 

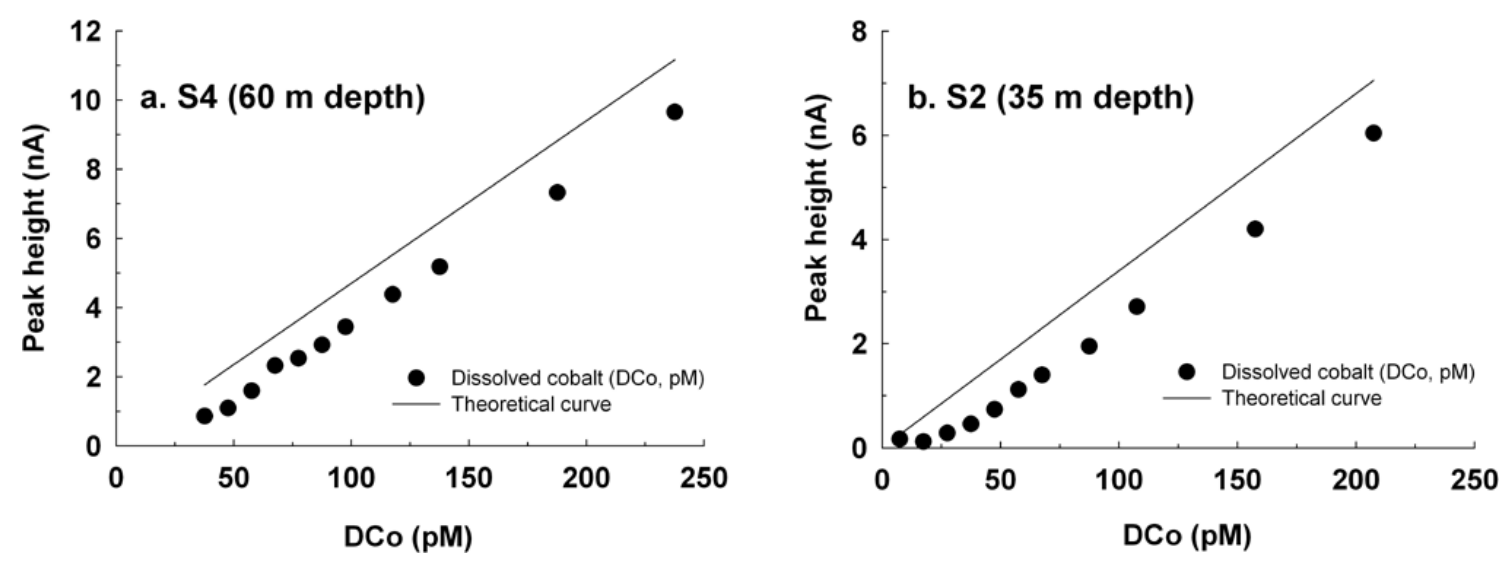

Fig. 6. Example of titration curves of the organic Co-binding ligands by standard additions of DCo (e.g.; peak height of the reduction current of Co-nioxime complexes as a function of cumulating DCo) illustrating the lack of curvature (titration a, left panel) and the curvature (titration b, right panel). The line represents the theoretical curve if no ligand was present in the sample. The lack of curvature below the theoretical curve (left panel) suggests that the organic ligands were initially saturated with Co, whereas the curvature (right panel) indicates that free Co-binding sites of organic ligands were titrated (see text for details).
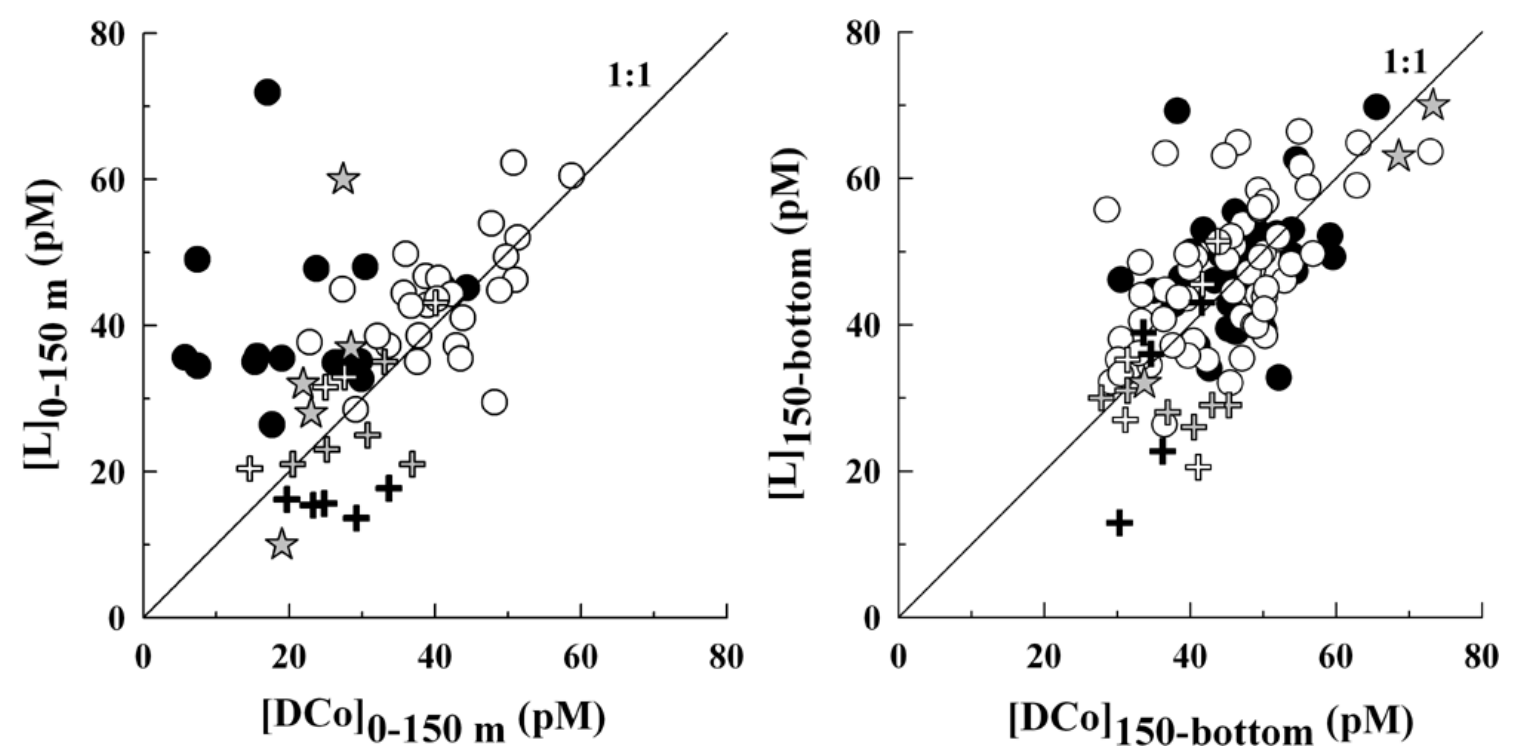

Fig. 7. Ocean data compilation of organic Co-binding ligands (L) versus dissolved cobalt (DCo) concentrations in the top $150 \mathrm{~m}$ ocean waters (right panel), and in the deep waters below $150 \mathrm{~m}$ depth (left panel).

Grey crosses: northeastern Atlantic (Ellwood and van den Berg, 2001); Black (north of PF) and white (south of PF) crosses: Southern Ocean (Ellwood et al., 2005); Grey stars: Sargasso Sea (Saito et al., 2001); Black circles: subtropical southeastern Atlantic and northern subantarctic domain (this study), White circles: Southern Ocean and northern Weddell Gyre (this study). The solid line represents the 1:1 line.

was no statistically significant correlation between $[\mathrm{L}]$ and [DCo] $\left(r^{2}=0.316, n=119\right)$ when all data available worldwide were considered (Fig. 7). However, we report L concentrations that are overall higher than or in the same range as DCo considering our whole dataset. This trend is also observed in other oceanic regions except at some depths in the deep North Atlantic (Ellwood and van den Berg, 2001) and north of the Polar Front (Ellwood et al., 2005) (Fig. 7).
In the present study, conditional stability constants are 1 to 5 orders of magnitude higher $\left(10^{17.9}\right.$ to $\left.10^{20.3}\right)$ than those previously reported elsewhere $\left(10^{15.6}\right.$ to $10^{17.2}$; Ellwood and van den Berg, 2001; Ellwood et al., 2005; Saito and Moffett, 2001). The discrepancy can reflect differences in the chemical nature of the organic ligands, but it can also be due to differences in the analytical methods used, particularly in the choice of the competing synthetic ligand (nioxime versus dimethylglyoxime), and in the detection window. Ellwood 
and van den Berg (2001) used a three orders of magnitude lower detection window (centred on $\alpha^{\prime}$ CoNioxime2 $=10^{4.7}$ ) and a higher $\mathrm{pH}(9.1)$ than we did $\left(10^{7.6}\right.$ and 8.1 , respectively), allowing for the detection of weaker Co organic complexes than in our study. Comparison between studies is difficult since there is not yet an intercomparison standard for ligand concentration and stability constant determinations. Given that vitamin $\mathrm{B}_{12}$ appears to be a frequently discussed complexing ligand for DCo, perhaps a chelexed and UVdigested, vitamin $\mathrm{B}_{12}$-amended seawater sample of a predetermined concentration may be an option as a standard to use in future studies.

\section{Cycle of the organic ligands along the section}

a. In the southeastern Atlantic and at its confluence with the subantarctic domain

The sources and the cycle of the organic Co-binding ligands in the ocean are not well known (Saito et al., 2001, 2005, 2010). In the present study, the relative [L] maxima observed within or close to the Chl-a rich layers in the subtropical domain and the northern subantarctic zone (stations L1, S1, L2, S2; Fig. 3) suggested biological production of Co-binding ligands in those waters. Additionally, the relative decreases of [L] concomitant with relative minima of DCo (stations L1, S1 and S2; Fig. 3) suggest biological uptake of L, probably as $\mathrm{CoL}$ complexes, and/or their biological degradation. The highest $[\mathrm{L}] /[\mathrm{DCo}]$ ratio recorded in the section was found in the surface waters marked by the highest subsurface maximum in Chl- $a$ concentrations, where high [L] (up to $72 \mathrm{pM}$ ) and the strongest depletion of DCo were also observed (at L2 and S2; Figs. 3-4). Hence, the highest [L]/[DCo] ratio probably reflected biological production of ligands and uptake of DCo in those surface waters. Furthermore, a linear relationship between the integrated concentrations of Chl- $a$ and [L] was obtained in the upper $100 \mathrm{~m}$ of the subtropical and the northern subantarctic domains, which became stronger when stations L1 to S2 were considered: $\quad[\text { Chl }-a]_{\text {int. }} 0-100 \mathrm{~m}=0.004 *[\mathrm{~L}]_{\text {int. }} 0-100 \mathrm{~m}+$ $11.9\left(r^{2}=0.781, n=4\right)$, further suggesting that phytoplankton could be a significant source of organic Co-binding ligands. Taxonomy study using an inverted microscope showed that unidentified nanoflagellates $(<5 \mu \mathrm{m})$ and dinoflagellates (Gymnodinium spp.) were the most abundant phytoplankton groups in the subtropical domain (Beker and Boye, 2010). This domain also featured oligotrophic conditions (Le Moigne et al., 2012) and relatively high regenerated production (Joubert et al., 2011), which leads us to think that cyanobacteria may also have been present. Cyanobacteria are often too small to be recognized clearly in light-microscope studies. The cyanobacteria
Synechococcus sp. and Prochlorococcus sp., which often dominate the picophytoplankton assemblage in oligotrophic regions (Partensky et al., 1999; Zhang et al., 2008a), are able to produce organic Co-binding ligands under different $\mathrm{Co}$ (and zinc) growth conditions as inferred by incubation experiments (Saito et al., 2002, 2005). It has also been suggested that biological production by Prochlorococcus and Synechococcus sp. can be the main source of $\mathrm{L}$ in the surface waters of the Sargasso Sea (Saito and Moffett, 2001). Similarly, production by cyanobacteria may be sufficient to cause the relative maxima of $[\mathrm{L}]$ observed in the subtropical surface waters in the present study.

The chemical nature of $L$ is virtually unknown. However, cyanobacteria have been shown to synthetize vitamin $B_{12}$ (Bonnet et al., 2010) in which Co occurs as the central atom. Conditional stability constants of Co complexes with vitamin $\mathrm{B}_{12} \quad\left(\log \mathrm{K}^{\prime} \mathrm{Co-B} 12\right)$ and coenzyme $\mathrm{B}_{12}\left(\log \mathrm{K}^{\prime}{ }_{\mathrm{Co}-\mathrm{coB} 12}\right)$ of 16.4 and 15.5, respectively, have been reported using a detection window centred on $\log \alpha^{\prime}$ CoNioxime2 of 4.7 (Ellwood and van den Berg, 2001). The mean conditional stability constant we reported in the $0-150 \mathrm{~m}$ surface layer $\left(\log \mathrm{K}^{\prime}{ }_{\mathrm{CoL}}=18.8 \pm 0.59, n=19\right)$ at a much higher detection window (centred on $\log \alpha^{\prime}$ CoNioxime2 of 7.6) is 2 to 3 orders of magnitude higher than those of vitamin $\mathrm{B}_{12}$ and coenzyme $\mathrm{B}_{12}$ Co-complexes. It is possible that vitamin $\mathrm{B}_{12}$ or compounds of similar nature have stronger affinity for Co than it has been reported previously, such that they would have been detected in the present study. Hence vitamin $\mathrm{B}_{12}$ or compounds of similar nature may have accounted for a significant portion of the organic ligands detected in the subtropical surface waters.

The conditional stability constants of Co-L complexes in this domain were in the same ranges in deep waters $\left(\log \mathrm{K}^{\prime} \mathrm{CoL}=18.9 \pm 0.42, n=45\right)$ as in surface waters $\left(\log \mathrm{K}^{\prime} \mathrm{CoL}=18.8 \pm 0.59, n=19\right)$, suggesting vertical export of $L$ produced in surface waters and/or production in deep waters, with the $\mathrm{L}$ produced in deep waters having a chemical nature (or at least an affinity for Co) similar to those produced near the surface. Furthermore, the rather invariant $[\mathrm{L}] /[\mathrm{DCo}]$ ratio of $\sim 1 \mathrm{pM} \mathrm{pM}^{-1}$ in intermediate and deep waters in this domain (Fig. 4) suggests that similar sources and sinks were impacting the cycles of both the organic ligands and Co in the deep ocean. The deep distribution of DCo indicates also inputs from the margins of South Africa with lateral advection of enriched intermediate (I-AAIW) and deep (SE-NADW) waters to the southeastern Atlantic Ocean (Bown et al., 2011). In these water-masses the distribution of L closely followed the DCo distribution and the concentrations were in the same range (Fig. 3; Table 1). Peculiarly, relatively high [L] was observed in the 
cores of I-AAIW $(49.4 \pm 2.4 \mathrm{pM}, n=6)$ and SENADW $(51.0 \pm 3.9 \mathrm{pM}, n=7)$. This suggests that the South African margins could also be a source of organic Co-binding ligands.

b. In the Antarctic Circumpolar Current and the Weddell Gyre

The surface distribution of $\mathrm{L}$ shifted southward within the ACC domain, where L concentrations paralleled those of DCo in surface waters with no evidence of high production of L (Fig. 3). There was no sub-surface maxima of $\mathrm{L}$, and no significant relationship between vertically integrated $\mathrm{L}$ and $\mathrm{Chl} a$ concentrations in these surface waters, especially in the post-diatom-bloom area observed around the Antarctic Polar Front (Le Moigne et al., 2012). These observations argue against an intense biological source of $\mathrm{L}$ in this domain, either directly by $\mathrm{L}$ production by diatoms or other phytoplankton taxa, or indirectly due to cell lysis or bacterial degradation of the organic matter. These features contrast with the observations gathered south of the Polar Front at $20^{\circ} \mathrm{E}$ (Ellwood et al., 2005) which suggested an indirect production of $\mathrm{L}$ due to degradation of molecules released by phytoplankton, possibly after cell death (Ellwood et al., 2005). In fact the almost invariant $[\mathrm{L}] /[\mathrm{DCo}]$ ratio of $\sim 1$ recorded in the surface waters (Figs. 4-5) and the uniform distributions of $\mathrm{L}$ and DCo further reflect conservative behaviour (or a balance between sources and sinks) of both L and DCo. The conditional stability constants of Co-L complexes in the surface waters of this domain $\left(\log \mathrm{K}^{\prime} \mathrm{CoL}=18.6 \pm 0.32, n=45\right)$ were in the same range as those reported in the north of the section $\left(\log \mathrm{K}^{\prime} \mathrm{CoL}=18.8 \pm 0.59, n=19\right)$. The ligands can thus be of the same chemical nature in the surface waters of the whole section, whereas the nano- and picoplankton assemblages were dominated by different taxa (Beker and Boye, 2010), which might drive biological production of chemically different organic ligands.

Below the nutricline, [L] increased with depth at some stations (e.g., L6 and S4; Fig. 3), indicating possible regeneration of $\mathrm{L}$ by bacterial activity there. In intermediate and deep waters, the concentrations and distributions of $\mathrm{L}$ were similar to those of DCo (A-AAIW: $54.5 \pm 9.16 \mathrm{pM}, n=6$; A-UCDW: $46.7 \pm 9.02 \mathrm{pM}, n=4$; DP-UCDW: $46.5 \pm 9.15 \mathrm{pM}$, $n=10$; Fig. 3 ), which were also reflected by almost invariant $[\mathrm{L}] /[\mathrm{DCo}]$ ratio of $\sim 1 \mathrm{pM} \mathrm{pM}^{-1}$ (Fig. 4). Thus the deep cycles of $\mathrm{L}$ and DCo could have been impacted by similar sources and sinks, like in the deep waters of the northern section. DCo distributions and physical hydrodynamics indicate remineralisation and inputs from the Drake Passage and the southwestern Atlantic to the $0^{\circ}$ meridian along the fast eastward flow of the Antarctic Circumpolar Current in the cores of A-AAIW and A-UCDW north of the Polar Front, as well as in the DP-UCDW south of the Polar Front (Bown et al., 2011). Hence, both local regeneration and transport of ligands from lithogenic sources over long distances by watermasses circulation could be sources of $\mathrm{L}$ in deep waters.

\subsection{Possible implications for the bioavailability of cobalt}

Classically in growth experiments the addition of a synthetic ligand (EDTA, NTA or DTPA) controls the speciation of $\mathrm{Co}$, and it is assumed that only the inorganic $\mathrm{Co}^{2+}$ species not bound to the added synthetic ligand are available for biological uptake (Sunda and Hunstman, 1995). Although those experiments provide important parameters, such as half-saturation constants for $\mathrm{Co}^{2+}\left(K_{\mathrm{mCo} 2+}\right)$, which can be species specific (Sunda and Huntsman, 1995), they do not allow to study the bio-availability of organic (CoL) and inorganic (Co') Co species naturally present in the ocean. Considering that DCo was found to be predominantly organically bound in our survey, it is important to study the bioavailability of Co bound to natural organic ligands in situ. However, only one study has evidenced utilization of CoL by the cyanobacterium Prochlorococcus (Saito et al., 2002). The biological uptake of Co by cyanobacteria, in addition to uptake by nanoflagellates and dino-flagellates may have significantly accounted for the strong DCo depletion observed in surface waters of the southern subtropical domain and northern subantarctic zone (Bown et al., 2011). In those waters, there was evidence of biological removal of $\mathrm{CoL}$ at the depths of DCo uptake further suggesting that Co could have been taken up from the organic complexes by cyanobacteria, nano- and dino-flagellates.

The growth rates of the coastal cyanobacterium Synechococcus bacillaris or Prochlorococcus are severely Colimited at $\mathrm{Co}^{2+}$ concentrations below $3 \times 10^{-14} \mathrm{M}$ and $1 \times 10^{-13} \mathrm{M}$, respectively (Saito et al., 2002; Sunda and Huntsman, 1995). Thus the extremely low inorganic Co concentrations recorded in these waters (e.g.; [Co'] $=2 \times 10^{-20}$ $2 \times 10^{-18} \mathrm{M}$; Table 1) would have severely limited the growth of cyanobacteria, if the cells were unable to take up Co from CoL complexes. There is no $\mathrm{K}_{\mathrm{m}}$ value for Colimited growth of nanoflagellates and dino-flagellates available in the literature to evaluate any limitation hypothesis in these waters. In any event, cyanobacteria (which have a known capacity to produce $\mathrm{L}$ ) and nano- and dino-flagellates may be able to assimilate, directly or indirectly, $\mathrm{CoL}$ in the euphotic layer of the subtropical and northern subantarctic domains.

Mechanisms of uptake of $\mathrm{CoL}$ are not known but the strong complexes observed in this study associated with their relative slowness for exchanging Co suggest that they do not 
readily dissociate for inorganic Co uptake. The organic $\mathrm{CoL}$ complexes may not be available to some eukaryotic phytoplankton as previously suggested (Saito et al., 2004, 2005). On the other hand, organic Co can be directly bioavailable to some phytoplankton species, especially those with specialized uptake systems, such as cobalophore and siderophorelike compounds (Duckworth et al., 2009), similar to the acquisition of iron with siderophores (Maldonado and Price, 2001; Shaked et al., 2005). In such a pathway, organic complexation of Co would thus enhance its availability to species able to take up organic CoL complexes, and may also increase the residence time of this pool, for example by slowing down rates of $\mathrm{Co}(\mathrm{II})$ oxidation to less soluble inorganic Co(III) species (Moffett and Ho, 1996) in the biologically oxygenated surface waters.

In surface waters of the ACC domain and the Weddell Gyre, the relatively uniform distributions of DCo (Fig. 2) and $\mathrm{CoL}$ (Table 1) suggested a balance between the removal of $\mathrm{Co}$ and its sources. The Polar Front region was characterized by a spatially extensive post-diatom-bloom condition (Le Moigne et al., 2012). Diatoms dominated the phytoplankton biomass (Beker and Boye, 2010), and in the Weddell Gyre this assemblage contained degraded frustules with small or absent chloroplasts also suggesting a late stage of a relatively minor diatom bloom (Le Moigne et al., 2012). Nevertheless, it has been suggested that biological uptake, especially by diatoms, may not have been the dominant mechanism for removal of DCo from these surface waters (Bown et al., 2011), probably because diatoms need much less Co than iron and (to a lesser extent) zinc to grow (Sunda and Huntsman, 1995). Furthermore, the higher DCo concentrations in those surface waters, compared with those to the north, combined with the overall higher proportion of inorganic Co led to inorganic Co concentrations as high as $11 \mathrm{pM}$ in the post-diatom-bloom waters (Table 1). The inorganic Co concentrations (Co') recorded in these waters were thus higher than the half-saturation $\mathrm{Co}^{2+}$ constant $\left(K_{\mathrm{mCo} 2+}\right)$ reported for a coastal diatom Thalassiosira pseudonana $\left(K_{\mathrm{mCo}+}=\right.$ $3.6 \times 10^{-12} \mathrm{M}$; Sunda and Huntsman, 1995), which presumably has a higher metals demand than its oceanic counterparts. Thus inorganic Co levels were probably not limiting the growth of the diatoms in the Polar Front region. Even Phaeocystis antarctica, if present in those waters, would not have been limited by the inorganic Co concentrations (e.g.; $K_{\mathrm{mCo} 2+}=1.9 \times 10^{-13} \mathrm{M}$; Saito and Goepfert, 2008).

\subsection{Potential impacts of the organic speciation on the geochemistry of cobalt}

Benthic and sediment inputs, and transport from continental shelf and slope waters towards the open ocean, may all be important sources of trace metals to the deep ocean (Bowie et al., 2002; Elderfield et al., 1981; Noble et al., 2008; Zhang et al., 2008b), notably for DCo (Noble et al., 2008; Huang and Conte, 2009; Bown et al., 2011). Processes by which DCo is released from sediments, such as reductive processes in the sediments or dissolution of resuspended lithogenic particles in the overlying waters, as well as those by which it is advected to intermediate and deep ocean waters, are almost unknown. Previous sediment work has shown that cobalt could be recycled during dissolution and reprecipitation of sedimentary manganese oxides between sub-oxic and oxic sediments and pore waters (Heggie and Lewis, 1984). Furthermore reduction of $\mathrm{Co}$ (III) oxides from margin sediments and transport of the more soluble $\mathrm{Co}$ (II) in low oxygenated deep waters that intersect the margin are conceivable (Bown et al., 2011), like it has been proposed for manganese (Johnson et al., 1996). Deep Co inputs might also be stabilized as Co organic complexes, where the ligands are probably enhancing Co dissolution from the sediment rocks (Hausrath et al., 2009), which in turn generates high DCo concentrations in deep waters (Saito and Moffett, 2002). The advection of DCo from the margins of South Africa has been found in intermediate (I-AAIW) and deep (SE-NADW) waters in the subtropical domain (Bown et al., 2011). In those waters most DCo was organically bound (at 85->99.9\%; Table 1), suggesting that $\mathrm{Co}$ is transported from the lithogenic sources mainly as organic complexes. In addition, these intermediate and deep water masses were generally characterized by higher [Co'] $\left(\sim 1.0 \times 10^{-18}\right.$ to $\left.9.0 \times 10^{-12} \mathrm{M}\right)$ compared to the surface waters, suggesting that a small but at times significant fraction of DCo (up to $15 \%$ ) is also transported as inorganic complexes in these waters. Yet stabilization of oxidized $\mathrm{Co}$ (III) by organic complexation in these rather oxygenated waters (Table 2) appears to be the main process that permits DCo to remain in solution while it is advected from South Africa margins, it is possible that reduced inorganic $\mathrm{Co}(\mathrm{II})$ species still account for a smaller, but not trivial, fraction of the transported Co.

The main reservoir of DCo along the section was found in the low-oxygenated Upper Circumpolar Deep Waters (AUCDW and DP-UCDW, Fig. 2), due to remineralization and lithogenic inputs from the Drake Passage and the southwestern Atlantic along the fast eastward flow of the ACC (Bown et al., 2011). The organic complexes represented more than $75 \%$ of DCo in these waters (Table 1), which suggests that Co is transported predominantly as organic complexes. The redox state of the Co bound to these organic ligands is not known, but the rather low oxygen content of these water masses $\left(160-190 \mu \mathrm{mol} \mathrm{O} \mathrm{O}_{2} \mathrm{~kg}^{-1}\right.$ ) suggests that the Co(II) redox state may dominate. The complexation of the more soluble $\mathrm{Co}(\mathrm{II})$ with organic chelates would probably promote the stabilization of Co in these waters. Furthermore, inorganic Co often accounted for a significant fraction of DCo in those waters, up to $25 \%$ (Table 1). The reduction of Co(III) oxides from margin sediments at the intersection of those low-oxygenated water masses with margins and continental slopes of South America, the Drake Passage and the Antarctic Peninsula, and transport of the more soluble inorganic $\mathrm{Co}$ (II) species can conceivably account for the relatively 
Table 2. Water-masses origins, characteristics and depth ranges along the BONUS-GoodHope section.

\begin{tabular}{|c|c|c|c|}
\hline Water-mass & Origin & Characteristic(s) & Depth range $(\mathrm{m})$ \\
\hline \multicolumn{4}{|l|}{ North of the Agulhas Ridge (stations L1 to S2) } \\
\hline I-AAIW: Indian Antarctic Intermediate Water ${ }^{1}$ & Indian Ocean & salinity $\geq 34.3$ & $800-1200(\mathrm{~L} 1$ and S1) \\
\hline A-AAIW: Atlantic Antarctic Intermediate Water ${ }^{2}$ & $\begin{array}{l}\text { Subantarctic region } \\
\text { of the southwest } \\
\text { Atlantic }\end{array}$ & $\begin{array}{l}\text { salinity } \leq 34.3 \\
\mathrm{O}_{2}>250 \mu \mathrm{mol} \mathrm{kg}\end{array}$ & $600-1000$ (L2 and S2) \\
\hline A-UCDW: Atlantic Upper Circumpolar Deep Water ${ }^{3}$ & Southwest Atlantic & $\begin{array}{l}\text { oxygen minimum } \\
\left(160-180 \mu \mathrm{mol} \mathrm{kg}^{-1}\right)\end{array}$ & $1200-1500(\mathrm{~L} 2$ and S2) \\
\hline SE-NADW: North Atlantic Deep Water ${ }^{4-5}$ & $\begin{array}{l}\text { North Atlantic } \\
\text { "southeastern } \\
\text { route" }\end{array}$ & salinity $>34.8$ & $2000-3200$ \\
\hline AABW: Antarctic Bottom Waters ${ }^{6}$ & Weddell Sea & $\begin{array}{l}\text { high oxygen, low } \\
\text { temperature, low } \\
\text { salinity }\end{array}$ & Below 3200 \\
\hline \multicolumn{4}{|l|}{ South of the Agulhas Ridge (stations L3 to S5) } \\
\hline A-AAIW: Atlantic Antarctic Intermediate Water ${ }^{1}$ & $\begin{array}{l}\text { Subantarctic region } \\
\text { of the southwest } \\
\text { Atlantic }\end{array}$ & $\begin{array}{l}\text { salinity } \leq 34.3 \\
\mathrm{O}_{2}>250 \mu \mathrm{mol} \mathrm{kg}^{-1}\end{array}$ & $300-600$ (L3 to L5) \\
\hline A-UCDW: Atlantic Upper Circumpolar Deep Water ${ }^{2}$ & Southwest Atlantic & $\begin{array}{l}\text { oxygen minimum } \\
\left(160-180 \mu \mathrm{mol} \mathrm{kg}^{-1}\right)\end{array}$ & $1000-1500$ (L3 to L5) \\
\hline $\begin{array}{l}\text { DP-UCDW: Drake Passage Upper Circumpolar Deep } \\
\text { Water }^{3}\end{array}$ & Southern Ocean & $\begin{array}{l}\text { oxygen minimum } \\
\left(160-180 \mu \mathrm{mol} \mathrm{kg}^{-1}\right)\end{array}$ & $250-700$ (L6 to L7) \\
\hline SW-NADW: North Atlantic Deep Water ${ }^{4-5}$ & $\begin{array}{l}\text { North Atlantic } \\
\text { "southwestern } \\
\text { route" }\end{array}$ & oxygen maximum & $1500-3000(\mathrm{~L} 3$ to L5) \\
\hline AABW: Antarctic Bottom Waters ${ }^{6}$ & Weddell Sea & $\begin{array}{l}\text { high oxygen, low } \\
\text { temperature, low } \\
\text { salinity }\end{array}$ & $\begin{array}{l}\text { Below } 3500(\mathrm{~S} 3) \text { below } \\
3000(\mathrm{~S} 5)\end{array}$ \\
\hline
\end{tabular}

${ }^{1}$ Gordon et al., 1992; ${ }^{2}$ Piola and Gordon, $1989 ;{ }^{3}$ Withworth and Nowlin, 1987; ${ }^{4}$ Arhan et al., 2003; ${ }^{5}$ Gladyshev et al., 2008; 6 Reid, 1989.

elevated DCo concentrations, in addition to the remineralisation of DCo as Co(II) species (Bown et al., 2011). The low temperatures may have also promoted stabilization of DCo as inorganic $\mathrm{Co}(\mathrm{II})$ species by slowing down its oxidation kinetics into more insoluble inorganic Co(III) species (Lee and Fisher, 1993; Moffett and Ho, 1996).

Finally, bottom enrichment of DCo in the Antarctic bottom waters was also evident, with increasing concentration along the water-mass pathway (Bown et al., 2011). This may result from sediment resuspension and/or mixing with North Atlantic deep waters in the Cape Basin. Similar trends of northward-increasing concentrations were observed for the organic ligands (Fig. 3) and organic Co (Table 1), and Co was more than $90 \%$ organically complexed in those waters. This indicates that DCo from bottom sediments could be stabilized and transported predominantly as organic complexes, in addition to suggesting that bottom sediments may be a source of Co chelators. Organic complexation may thus prevent Co from being further scavenged after it has been released from bottom sediments.

\section{Summary and conclusion}

The distribution of the organic Co-binding ligands suggests a biological source, potentially driven by cyanobacteria, in the subtropical domain and the northern subantarctic zone. In contrast, a rather conservative behaviour was observed in the central and south Antarctic Circumpolar Current (ACC) and the Weddell Gyre, which may reflect a balance between production and removal of Co-binding ligands. Dissolved cobalt occurred predominantly as organic complexes along the section. The production of Co-binding ligands may have enhanced the bioavailability of DCo in the subtropical waters 
to phytoplankton species with specialized $\mathrm{CoL}$ acquisition pathways, although mechanisms of organic Co uptake still need to be addressed. Biological uptake of organic Co would explain the nutrient like distribution of DCo observed in these waters (Bown et al., 2011). In surface waters of the ACC and Weddell Gyre inorganic Co concentrations were not at limiting levels, even though inorganic species were present at much lower concentrations than organic Co. In intermediate and deep waters, remineralization and advection of water masses that have been in contact with continental margins were sources of DCo (Bown et al., 2011), as well as organic ligands. The processes by which Co is released from the sediments (both reductive and non-reductive) need further investigation, yet this study has shown that sedimentary Co inputs are predominantly as organic complexes. The complexation of Co with organic ligands may have promoted the stabilization of $\mathrm{Co}$ in the dissolved fraction, thus increasing its residence time over large scale of water-masses circulation.

Reduction of Co(III) oxides from margin sediments and transport of the more soluble inorganic $\mathrm{Co}(\mathrm{II})$ in the low oxygenated Upper Circumpolar Deep Waters may also be significant. Relatively high DCo concentrations in intermediate and deep waters could thus reflect sedimentary inputs, especially in the low oxygenated deep waters that intersect continental margins where the signature of these inputs was marked by a relative higher proportion of inorganic Co. More studies of the organic speciation of Co would be required to understand the interactions of DCo with the phytoplankton communities, as well as the use of DCo as a potential tracer of lithogenic sources in the intermediate and deep ocean.

Acknowledgements. We are most grateful for the assistance and the wonderful work at sea provided by Captain P. Courtés, the officers and the crew members of the French research vessel Marion Dufresne II during the IPY MD166 BONUS-GoodHope cruise. The chief scientists, M. Boye and S. Speich, and their collaborators M. Arhan (LPO, Brest, FR) and F. Dehairs (VUB, Brussels, Belgium) are warmly acknowledged for their efforts. We are grateful to the colleagues of our GO-FLO sampling team (F. Chever, B. Wake, E. Buciarrelli, G. Sarthou, F. Lacan and A. Radic). This investigation was financed by the BONUS-GoodHope project and cruise funded by the French LEFE National Program of INSU, the National Agency of French Research (ANR-07-BLAN-0146-01) and the Paul Emile Victor Institut. This work is a contribution to the International Polar Year and the International GEOTRACES programmes. The Region Bretagne supported the $\mathrm{PhD}$ fellowship of J. B.

Edited by: F. Dehairs

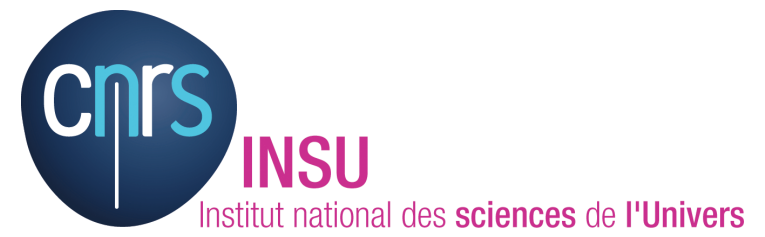

The publication of this article is financed by CNRS-INSU.

\section{References}

Arhan, M., Mercier, H., and Park, Y. H.: On the deep water circulation of the eastern South Atlantic Ocean, Deep-Sea Res. Pt. I, 50, 889-916, 2003.

Beker, B. and Boye, M.: Phytoplankton assemblages in the Southern Ocean during the International Polar Year, Ocean Sci. Meeting, 22-26 February 2010, Portland, USA, 2010.

Belkin, I. M. and Gordon, A. L.: Southern Ocean fronts from the Greenwich meridian to Tasmania, J. Geophys. Res.-Oceans, 101, 3675-3696, 1996.

Bertrand, E. M., Saito, M. A., Rose, J. M., Riesselman, C. R., Lohan, M. C., Noble, A. E., Lee, P. A., and DiTullio, G. R.: Vitamin B-12 and iron colimitation of phytoplankton growth in the Ross Sea, Limnol. Oceanogr., 52, 1079-1093, 2007.

Bonnet, S., Webb, E. A., Panzeca, C., Karl, D. M., Capone, D. G., and Sanudo-Wilhelmy, S. A.: Vitamin $\mathrm{B}_{12}$ excretion by cultures of the marine cyanobacteria Crocosphaera and Synechococcus, Limnol. Oceanogr., 55, 1959-1964, 2010.

Bowie, A. R., Whitworth, D. J., Achterberg, E. P., Mantoura, R. F. C., and Worsfold, P. J.: Biogeochemistry of Fe and other trace elements (Al, Co, Ni) in the upper Atlantic Ocean, Deep-Sea Res. Pt. I, 49, 605-636, 2002.

Bown, J., Boye, M., Baker, A., Duvieilbourg, E., Lacan, F., Le Moigne, F., Planchon, F., Speich, S., and Nelson, D. M.: The biogeochemical cycle of dissolved cobalt in the Atlantic and the Southern Ocean south off the coast of South Africa, Mar. Chem., 126, 193-206, 2011.

Duckworth, O. W., Bargar, J. R., Jarzecki, A. A., Oyerinde, O., Spiro, T. G., and Sposito, G.: The exceptionally stable cobalt(III)-desferrioxamine B complex, Mar. Chem., 113, 114122, 2009.

Elderfield, H., McCaffrey, R. J., Luedtke, N., Bender, M., and Truesdale, V. W.: Chemical Diagenesis in Narragansett Bay Sediments, Am. J. Sci., 281, 1021-1055, 1981.

Ellwood, M. J. and van den Berg, C. M. G.: Determination of organic complexation of cobalt in seawater by cathodic stripping voltammetry, Mar. Chem., 75, 33-47, 2001.

Ellwood, M. J., van den Berg, C. M. G., Boye, M., Veldhuis, M., de Jong, J. T. M., de Baar, H. J. W., Croot, P. L., and Kattner, G.: Organic complexation of cobalt across the Antarctic Polar Front in the Southern Ocean, Mar. Freshwater Res., 56, 1069-1075, 2005.

Gladyshev, S., Arhan, M., Sokov, A., and Speich, S.: A hydrographic section from South Africa to the southern limit of the Antarctic Circumpolar Current at the Greenwich meridian, DeepSea Res. Pt. I, 55, 1284-1303, 2008.

Gordon, A. L., Weiss, R. F., Smethie, W. M., and Warner, M. J.: Thermocline and Intermediate Water Communication between 
the South-Atlantic and Indian Oceans, J. Geophys. Res.-Oceans, 97, 7223-7240, 1992.

Gordon, G. T. and Sullivan, C. W.: Vitamin $\mathrm{B}_{12}$ and cobalt cycling among diatoms and bacteria in Antarctica sea ice microbial communities, Limnol. Oceanogr., 53, 1862-1877, 2008.

Guéneuguès, A. and Boye, M.: Chlorophyll-a and phaeopigments along BONUS-GoodHope section. Cruise report of MD166 BONUS-GoodHope in Biogeochemistry and Geochemistry, Boye, M. and the cruise participants, 2008 (135 pp.), 108-110, 2008.

Hausrath, E. M., Neaman, A., and Brantley, S. L.: Elemental release rates from dissolving basalt and granite with and without organic ligands, Am. J. Sci., 309, 633-660, 2009.

Heggie, D. and Lewis, T.: Cobalt in pore waters of marine sediments, Nature, 311, 453-455, 1984.

Huang, S. and Conte, M. H.: Source/process apportionment of major and trace elements in sinking particles in the Sargasso sea, Geochim. Cosmochim. Acta, 73, 65-90, 2009.

Jakuba, R. W., Moffett, J. W., and Dyhrman, S. T.: Evidence for the linked biogeochemical cycling of zinc, cobalt, and phosphorus in the western North Atlantic Ocean, Global Biogeochem. Cy., 22, GB4012, doi:10.1029/2007GB003119, 2008.

Johnson, K. S., Coale, K. H., Berelson, W. M., and Gordon, R. M.: On the formation of the manganese maximum in the oxygen minimum, Geochim. Cosmochim. Acta, 60, 1291-1299, 1996.

Joubert, W. R., Thomalla, S. J., Waldron, H. N., Lucas, M. I., Boye, M., Le Moigne, F. A. C., Planchon, F., and Speich, S.: Nitrogen uptake by phytoplankton in the Atlantic sector of the Southern Ocean during late austral summer, Biogeosciences, 8, $2947-$ 2959, doi:10.5194/bg-8-2947-2011, 2011.

Knauer, G. A., Martin, J. H., and Gordon, R. M.: Cobalt in Northeast Pacific Waters, Nature, 297, 49-51, 1982.

Le Moigne, F. A. C., Boye, M., Masson, A., Corvaisier, R., Grossteffan, E., Guéneugues, A., and Pondaven, P.: Description of the biogeochemical features of the subtropical southeastern Atlantic and the Southern Ocean south off South Africa during the austral summer of the International Polar Year, Biogeosciences Discuss., 9, 5011-5048, doi:10.5194/bgd-9-5011-2012, 2012.

Lee, B. G. and Fisher, N. S.: Microbially mediated cobalt oxidation in seawater revealed by radiotracer experiments, Limnol. Oceanogr., 38, 1593-1602, 1993.

Maldonado, M. T. and Price, N. M.: Reduction and transport of organically bound iron by Thalassiosira oceanica (Bacillariophyceae), J. Phycol., 37, 298-309, 2001.

Martell, A. E. and Smith, R. M.: Critical stability constants, Plenum Press, New York, 495 pp., 1977.

Menzel, D. W. and Spaeth, J. P.: Occurrence of vitamin B $_{12}$ in the Sargasso Sea, Limnol. Oceanogr., 7, 151-154, 1962.

Moffett, J. W. and Ho, J.: Oxidation of cobalt and manganese in seawater via a common microbially catalyzed pathway, Geochim. Cosmochim. Acta, 60, 3415-3424, 1996.

Morel, F. M. M., Milligan, A. J., Saito, M. A., Heinrich, D. H., and Karl, K. T.: Marine Bioinorganic Chemistry: The Role of Trace Metals in the Oceanic Cycles of Major Nutrients, Treatise on Geochemistry, Pergamon, Oxford, 113-143, 2003.

Noble, A. E., Saito, M. A., Maiti, K., and Benitez-Nelson, C. R.: Cobalt, manganese, and iron near the Hawaiian Islands: A potential concentrating mechanism for cobalt within a cyclonic eddy and implications for the hybrid-type trace metals, Deep-Sea Res.
Pt. II, 55, 1473-1490, 2008.

Orsi, A. H., Whitworth, T., and Nowlin, W. D.: On the Meridional Extent and Fronts of the Antarctic Circumpolar Current, DeepSea Res. Pt. I, 42, 641-673, 1995.

Partensky, F., Hess, W. R., and Vaulot, D.: Prochlorococcus, a marine photosynthetic prokaryote of global significance, Microbiol. Molecular Biol. Rev., 63, 106-127, 1092-2172/99/\$04.0010, 1999.

Piola, A. R. and Gordon, A. L.: Intermediate Waters in the Southwest South-Atlantic, Deep-Sea Res. Pt. I, 36, 1-16, 1989.

Reid, J. L.: On the Total Geostrophic Circulation of the SouthAtlantic Ocean - Flow Patterns, Tracers, and Transports, Progr. Oceanogr., 23, 149-244, 1989.

Ruzic, I.: Theoretical aspects of the direct titration of natural waters and its information yield for trace-metal speciation, Anal. Chim. Acta, 140, 99-113, 1982.

Saito, M. A. and Moffett, J. W.: Complexation of cobalt by natural organic ligands in the Sargasso Sea as determined by a new highsensitivity electrochemical cobalt speciation method suitable for open ocean work, Mar. Chem., 75, 49-68, 2001.

Saito, M. A. and Goepfert, T. J.: Zinc-cobalt colimitation of Phaeocystis antarctica, Limnol. Oceanogr., 53, 266-275, 2008.

Saito, M. A., Moffett, J. W., Chisholm, S. W., and Waterbury, J. B.: Cobalt limitation and uptake in Prochlorococcus, Limnol. Oceanogr., 47, 1629-1636, 2002.

Saito, M. A., Moffett, J. W., and DiTullio, G. R.: Cobalt and nickel in the Peru upwelling region: A major flux of labile cobalt utilized as a micronutrient, Global Biogeochem. Cy., 18, GB4030, doi:10.1029/2003GB002216, 2004.

Saito, M. A., Rocap, G., and Moffett, J. W.: Production of cobalt binding ligands in a Synechococcus feature at the Costa Rica upwelling dome, Limnol. Oceanogr., 50, 279-290, 2005.

Saito, M. A., Goepfert, T. J., Noble, A. E., Bertrand, E. M., Sedwick, P. N., and DiTullio, G. R.: A seasonal study of dissolved cobalt in the Ross Sea, Antarctica: micronutrient behavior, absence of scavenging, and relationships with $\mathrm{Zn}, \mathrm{Cd}$, and P, Biogeosciences, 7, 4059-4082, doi:10.5194/bg-7-4059-2010, 2010.

Schlitzer, R.: Ocean Data View, http://odv.awi.de., 2012.

Shaked, Y., Kustka, A. B., and Morel, F. M. M.: A general kinetic model for iron acquisition by eukaryotic phytoplankton, Limnol. Oceanogr., 50, 872-882, 2005.

Sunda, W. G. and Huntsman, S. A.: Cobalt and zinc interreplacement in marine phytoplankton: Biological and geochemical implications, Limnol. Oceanogr., 40, 1404-1417, 1995.

Turner, D. R., Whitfield, M., and Dickson, A. G.: The equilibrium speciation of dissolved components in freshwater and seawater at 25 degrees $\mathrm{C}$ and $1 \mathrm{~atm}$ pressure, Geochim. Cosmochim. Acta, 45, 855-881, 1981.

van den Berg, C. M. G.: Determination of copper complexation with natural organic-ligands in sea-water by equilibration with $\mathrm{MnO}_{2}$, Mar. Chem., 11, 307-322, 1982.

van den Berg, C. M. G. and Kramer, J. R.: Determination of complexing capacities of ligands in natural-waters and conditional stability-constants of the copper-complexes by means of manganese-dioxide, Anal. Chim. Acta, 106, 113-120, 1979.

Whitworth, T. and Nowlin, W. D.: Water Masses and Currents of the Southern-Ocean at the Greenwich Meridian, J. Geophys. Res.Oceans, 92, 6462-6476, 1987. 
Xue, Y. and Traina, S.: Oxidation kinetics of Co(II)-EDTA in aqueous and semi-aqueous goethite suspensions, Environ. Sci. Technol., 30, 1975-1981, 1996.

Yang, R. J. and Van den Berg, C. M. G.: Metal complexation by humic substances in seawater, Environ. Sci. Technol., 43, 71927197, 2009.

Zhang, H., Van den Berg, C. M. G., and Wollast, R.: The determination of interactions of cobalt (II) with organic compounds in seawater using cathodic stripping voltammetry, Mar. Chem., 28, 285-300, 1990.
Zhang, Y., Jiao, N. Z., and Hong, N.: Comparative study of picoplankton biomass and community structure in different provinces from subarctic to subtropical oceans, Deep-Sea Res. Pt. II, 55, 1605-1614, 2008a.

Zhang, Y., Lacan, F., and Jeandel, C.: Dissolved rare earth elements tracing lithogenic inputs over the Kerguelen Plateau (Southern Ocean), Deep-Sea Res. Pt. II, 55, 638-652, 2008 b. 